\title{
$\omega$ QRB-Domains and the Probabilistic Powerdomain
}

\author{
Jean Goubault-Larrecq ${ }^{\dagger, *}$ \\ † Preuves, Programmes et Systèmes, UMR 7126, CNRS and University Paris Diderot \\ * LSV, ENS Cachan, CNRS, INRIA
}

\begin{abstract}
Is there any cartesian-closed category of continuous domains that would be closed under Jones and Plotkin's probabilistic powerdomain construction? This is a major open problem in the area of denotational semantics of probabilistic higher-order languages. We relax the question, and look for quasi-continuous dcpos instead. We introduce a natural class of such quasi-continuous dcpos, the $\omega \mathbf{Q R B}$-domains. We show that they form a category $\omega$ QRB with pleasing properties: $\omega \mathrm{QRB}$ is closed under the probabilistic powerdomain functor, has all finite products, all bilimits, and is stable under retracts, and even under so-called quasiretracts. But... $\omega$ QRB is not cartesian closed.
\end{abstract}

\section{Introduction}

Write $\mathbf{V}_{1}(X)$ (resp. $\left.\mathbf{V}_{\leq 1}(X)\right)$ the depo of all continuous probability (resp., subprobability) valuations over $X$, and call it the probabilistic (resp., subprobabilistic) powerdomain of $X$. This construction was introduced by Jones and Plotkin to give a denotational semantics to higher-order probabilistic languages [13]—we define it precisely later.

A famous open problem in the area is whether the probabilistic powerdomain of an FS-domain is again an FSdomain [16], and similarly with RB-domains in lieu of FSdomains. More generally, is there a category of nice enough dcpos that would be cartesian-closed and closed under $\mathbf{V}_{1}$ ? We call this the Jung-Tix problem. By "nice enough", we mean nice enough to do any serious mathematics with, e.g., to establish definability or full abstraction results in extensional models of higher-order, probabilistic languages. It is traditional to equate "nice enough" with "continuous", and this is justified by the rich theory of continuous domains [7].

However, quasi-continuous dcpos (see [8], or [7, III3]) generalize continuous dcpos and are almost as wellbehaved. We propose to widen the scope of the problem, and ask for a category of quasi-continuous dcpos that would be closed under $\mathbf{V}_{1}$. We show that, by mimicking the construction of RB-domains [1], with some flavor of "quasi",
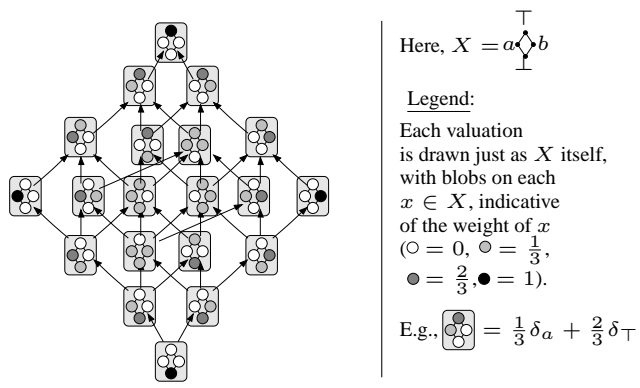

Figure 1. Part of the Hasse Diagram of $\mathrm{V}_{1}(X)$

we obtain a category $\omega \mathbf{Q R B}$ of so-called $\omega \mathbf{Q R B}$-domains that not only has many desired, nice mathematical properties (e.g., it is closed under bilimits, and every $\omega$ QRBdomain is stably compact), but is also closed under $\mathbf{V}_{1}$.

We in fact failed to solve the Jung-Tix problem: $\omega$ QRB is indeed not cartesian-closed. In spite of this, we believe our contribution to bring some progress towards settling the question, and at least to understand the structure of $\mathbf{V}_{1}(X)$ better. To appreciate this, recall what is currently known about $\mathbf{V}_{1}$. There are two landmark results: $\mathbf{V}_{1}(X)$ is a continuous depo as soon as $X$ is ([6], building on Jones [13]), and $\mathbf{V}_{1}(X)$ is stably compact (with its weak topology) whenever $X$ is $[16,2]$. Since then, no significant progress has been made. When it comes to solving the Jung-Tix problem, we must in fact realize that there is little choice: the only known cartesian-closed categories of (pointed) continuous dcpos that may suit our needs are RB and FS [16]. I.e., all other known cartesian-closed categories of continuous dcpos, e.g., bc-domains or L-domains, are not closed under $\mathbf{V}_{1}$. Next, we must recognize that little is known about the (sub)probabilistic powerdomain of an $\mathbf{R B}$ or $\mathbf{F S}$-domain. In trying to show that either $\mathbf{R B}$ or FS was closed under $\mathbf{V}_{1}$, Jung and Tix [16] only managed to show that the subprobabilistic powerdomain $\mathbf{V}_{\leq 1}(X)$ of a finite tree $X$ was an RB-domain, and that the subprobabilistic powerdomain of a reversed finite tree was an FSdomain. This is still far from the goal.

In fact, we do not know whether $\mathbf{V}_{1}(X)$ is an $\mathbf{R B}$ - 


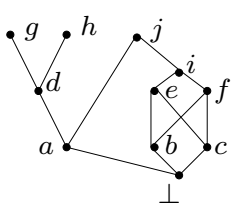

(i) A finite poset

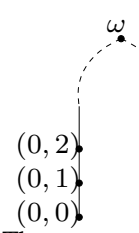

$(0,0)$.

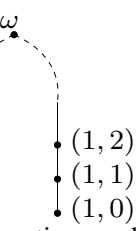

- $(1,0)$

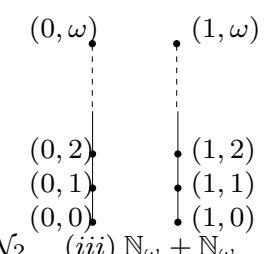

Figure 2. Poset Examples

domain when $X$ is even the simple poset $\{\perp, a, b, \top\}$ ( $a$ and $b$ incomparable, $\perp \leq a, b \leq \top$, see Figure 1, right) but it is an FS-domain. For a more complex (arbitrarily chosen) example, take $X$ to be the finite pointed poset of Figure $2(i)$ : then $\mathbf{V}_{1}(X)$ and $\mathbf{V}_{\leq 1}(X)$ are continuous and stably compact, but not known to be RB-domains or FSdomains (and they are much harder to visualize, too).

No progress seems to have been made on the question since Jung and Tix' 1998 attempt. As part of our results, we show that for every finite pointed poset $X$, e.g. Figure $2(i)$, $\mathbf{V}_{1}(X)$ is a continuous $\omega \mathbf{Q R B}$-domain. This is also one of the basic results that we then leverage to show that $\mathbf{V}_{1}(X)$ is an $\omega \mathbf{Q R B}$-domain for any $\omega \mathbf{Q R B}$-domain, in particular every RB-domain, not just every finite pointed poset, $X$.

One may obtain some intuition as to why this should be so, and at the same time give an idea of what $(\omega)$ QRBdomains are. Let $X$ be a finite pointed poset. In attempting to show that $\mathbf{V}_{1}(X)$ is an $\mathbf{R B}$-domain, we are led to study the so-called deflations $f: \mathbf{V}_{1}(X) \rightarrow \mathbf{V}_{1}(X)$, i.e., the continuous maps $f$ with finite range such that $f(\nu) \leq \nu$ for every continuous probability $\nu$ valuation on $X$, and we must try to find deflations $f$ such that $f(\nu)$ is as close as one desires to $\nu$. All natural definitions of $f$ fail to be continuous, and in fact to be monotonic. (E.g., Graham's construction [12] is not monotonic, see Jung and Tix.) Looking for maps $f$ such that $f(\nu)$ is instead a finite, non-empty set of valuations below $\nu$ shows more promise - the monotonicity requirements are slightly more relaxed. Such a set-valued function is what we call a quasi-deflation below. For example, one may think of fixing $N \geq 1$ ( $N=3$ in Figure 1), and mapping $\nu$ to the collection of all valuations $\nu^{\prime}$ below $\nu$ such that the measure of any subset is a multiple of $1 / N$, keeping only those $\nu^{\prime}$ that are maximal. (Pick them from the left of Figure 1, in our example.) This still does not provide anything monotonic, but we managed to show that one can indeed approximate every element $\nu$ of $\mathbf{V}_{1}(X)$, continuously in $\nu$, using quasi-deflations. The proof is non-trivial, and rests on deep properties relating QRB-domains and quasi-retractions - all notions that we define and study.

Outline. We introduce most of the required notions in Section 2. Since we shall only start studying the probabilistic powerdomain in Section 6, we shall refrain from defining valuations, probabilities, and related concepts until then. We introduce QRB-domains in Section 3. They are defined just as RB-domains are, only with a flavor of "quasi", i.e., replacing approximating elements by approximating sets of elements. We establish their main properties there, in particular that they are quasi-continuous, stably compact, and Lawson-compact. Much as RB-domains are also characterized as the retracts of bifinite domains, we show that, up to a few details, the QRB-domains are the quasi-retracts of bifinite domains in Section 4. Quasiretractions are an essential concept in the study of QRBdomains, as well as the close notion of quasi-projectiontwo notions that we introduce. We also show that the category of countably based QRB-domains is closed under finite products (easy) and bilimits (hard, but similar to the case of RB-domains) in Section 5. The core of the paper is Section 6, where we show that the category $\omega \mathbf{Q R B}$ of countably based QRB-domains is closed under the probabilistic powerdomain construction. This capitalizes on all previous sections, and will follow from Jung and Tix' result that $\mathbf{V}_{1}(X)$ is an $\mathbf{R B}$-domain whenever $X$ is a finite tree, and applying suitable quasi-projections and bilimits. The key result will then be Theorem 6.1, which shows that for any quasi-projection $Y$ of a stably compact space $X$, $\mathbf{V}_{1}(Y)$ is again a quasi-projection of $\mathbf{V}_{1}(X)$, again up to a few details. We conclude in Section 7.

Other Related Work. Instead of solving the Jung-Tix problem, one may try to circumvent it. One of the most successful such attempts led to the discovery of qcb-spaces [4] and to compactly generated countably based monotone convergence spaces [3], as cartesian-closed categories of topological spaces where a reasonable amount of semantics can be done. This provides exciting new perspectives. The category of qcb-spaces accommodates two probabilistic powerdomains [5]. The observationally induced one is essentially $\mathbf{V}_{1}(X)$ (with the weak topology), but differs from the one obtained as a free algebra. Since the latter is essentially the least one containing all finite linear combination of Dirac masses $\sum_{i=1} a_{i} \delta_{x_{i}}$, it would be the preferred model to establish a definability result: if each $x_{i}$ is definable, then $\sum_{i=1} a_{i} \delta_{x_{i}}$ is the distribution one gets by picking each $x_{i}$ with probability $a_{i}$, which is easy to do by program. Unfortunately, it is the other, observationally induced model that seems to enjoy nice mathematical properties.

\section{Preliminaries}

We refer to $[1,7,20]$ for background material. A poset $X$ is a set with a partial ordering $\leq$. Let $\downarrow A$ be the downward closure $\{x \in X \mid \exists y \in A \cdot x \leq y\}$; we write $\downarrow x$ for $\downarrow\{x\}$, when $x \in X$. The upward closures $\uparrow A, \uparrow x$ are defined similarly. When $x \leq y, x$ is below $y$ and $y$ is above $x$. $X$ is pointed iff it has a least element $\perp$. A $d c p o$ is a poset $X$ where every directed family $\left(x_{i}\right)_{i \in I}$ has a least upper bound $\sup _{i \in I} x_{i}$; directedness means that $I \neq \emptyset$ and for 
every $i, i^{\prime} \in I$, there is an $i^{\prime \prime} \in I$ such that $x_{i}, x_{i^{\prime}} \leq x_{i^{\prime \prime}}$.

Every poset $X$ comes with a topology, whose opens $U$ are the upward closed subsets such that, for every directed family $\left(x_{i}\right)_{i \in I}$ that has a least upper bound in $U, x_{i} \in U$ for some $i \in I$. This is the Scott topology. When we see a poset or dcpo $X$ as a topological space, we will implicitly assume the latter, unless marked otherwise.

There is a deep connection between order and topology. Given any topological space $X$, its specialization quasiordering $\leq$ is defined by $x \leq y$ iff every open containing $x$ also contains $y$. The specialization quasi-ordering of a dcpo $X$ (with ordering $\leq$, and equipped with its Scott topology), is the original ordering $\leq$.

A subset $A$ of a topological space $X$ is saturated iff it is the intersection of all opens $U$ containing $A$. Equivalently, $A$ is upward closed in the specialization quasi-ordering [20, Remark after Definition 4.34]. So we can, and shall often prove inclusions $A \subseteq B$ where $B$ is upward closed by showing that every open $U$ containing $B$ also contains $A$.

A map $f: X \rightarrow Y$ between topological spaces is continuous iff $f^{-1}(V)$ is open for every open subset $V$ of $Y$. Every continuous map is monotonic with respect to the underlying specialization quasi-orderings. When $X$ and $Y$ are posets, it is equivalent to require $f$ to be Scott-continuous, i.e., to be monotonic and to preserve existing directed least upper bounds. A homeomorphism if a bijective continuous map whose inverse is also continuous.

Given a set $X$, and a family $\mathcal{B}$ of subsets of $X$, there is a smallest topology containing $\mathcal{B}$ : then $\mathcal{B}$ is a subbase of the topology, and its elements are the subbasic opens. To show that $f: X \rightarrow Y$ is continuous, it is enough to show that the inverse image of every subbasic open of $Y$ is open in $X$.

The interior $\operatorname{int}(A)$ of a subset $A$ of a topological space $X$ is the largest open contained in $A$. A subset $Q$ of a topological space $X$ is compact iff one can extract a finite subcover from every open cover of $Q$. The important ones are the saturated compacts. For any finite subset $A, \uparrow A$ is compact and saturated; we call such special saturated compacts the finitary compacts. $X$ is locally compact iff for each open $U$ and each $x \in U$, there is a compact saturated subset $Q$ such that $x \in \operatorname{int}(Q)$ and $Q \subseteq U$. X is sober iff every irreducible closed subset is the closure of a unique point; in the presence of local compactness (and when $X$ is $T_{0}$, i.e., when the specialization quasi-ordering is an ordering), it is equivalent to require that $X$ be well-filtered [7, Theorem II-1.21], i.e., to require that, for every open $U$, for every filtered family $\left(Q_{i}\right)_{i \in I}$ of saturated compacts such that $\bigcap_{i \in I}^{\downarrow} Q_{i} \subseteq U, Q_{i} \subseteq U$ for some $i \in I$ already. We say that the family is filtered iff it is directed in the $\supseteq$ ordering, and make it explicit by using $\downarrow$ as superscript. (Symmetrically, we write $\bigcup^{\uparrow}$ for directed unions.)

Given any poset $X$, any finite subset $E$ of $X$, and any element $x$ of $X$, we write $E \leq x$ iff $x \in \uparrow E$, i.e., iff there is a $y \in E$ such that $y \leq x$. E approximates $x$, in notation $E \ll x$, iff for every directed family $\left(x_{i}\right)_{i \in I}$ that has a least upper bound above $x$, then $E \leq x_{i}$ for some $i \in I$. (We shall also write $y \ll x$, when $y \in X$, as shorthand for $\{y\} \ll x$; this is the more familiar way-below relation. But beware that $E \ll x$ means that all elements of $E$ approximate $x$ collectively, while none in particular may approximate $x$ individually.) We compare finite subsets in the Smyth quasi-ordering $\leq^{\sharp}$, defined by $E<\sharp E^{\prime}$ iff $\uparrow E \supseteq \uparrow E^{\prime}$, or equivalently, for every $y^{\prime} \in E^{\prime}$, there is a $y \in E$ such that $y \leq y^{\prime}$. Two finite subsets $E$ and $E^{\prime}$ are equivalent iff $E \leq \sharp E^{\prime}$ and $E^{\prime} \leq \sharp E$, iff $\uparrow E=\uparrow E^{\prime}$. The collection of equivalence classes of non-empty finite subsets $E$ of $X$ is denoted by $\operatorname{Fin}(X)$. The quasi-ordering $\leq \sharp$ induces a partial ordering, again written $\leq^{\sharp}$, on $\operatorname{Fin}(X)$. We abuse notation and confuse finite subsets $E$ with their equivalence classes. Observe that if $E \leq^{\sharp} E^{\prime}$ and $E^{\prime} \ll x$, then $E \ll x$, so in particular the notation $E \ll x$ is independent of the choice of $E$ in its equivalence class.

A quasi-continuous dcpo $X$ (see [8] or [7, Definition III3.2]) is a dcpo such that, for every $x \in X$, the collection of all $E \in \operatorname{Fin}(X)$ such that $E \ll x$ is directed (w.r.t. $\leq^{\sharp}$ ) and $\bigcap_{E \in \operatorname{Fin}(X)} \uparrow E=\uparrow x$. The theory of quasi-continuous dcpos is less well explored than that of continuous dcpos, but quasi-continuous dcpos retain many of the properties of the latter. (Every continuous depo is quasi-continuous, but not conversely. A counterexample is given by $\mathcal{N}_{2}$, see Figure $2(i i)$.) Every quasi-continuous dcpo $X$ is locally compact and sober [7, III-3.7]. In a quasi-continuous dcpo $X$, for every $E \in \operatorname{Fin}(X)$, the set $\uparrow E$ defined as $\{x \in X$ $E \ll x\}$, is open, and equals $\operatorname{int}(\uparrow E)$ [7, III-3.6(ii)]; every open $U$ is the union of all the subsets $\uparrow E, E \in \operatorname{Fin}(X)$, contained in $U$ [7, III-5.6]; and for every compact saturated subset $Q$ and every open subset $U$ containing $Q$, there is a finite subset $E$ of $X$ such that $Q \subseteq \uparrow E$ and $E \subseteq U$ [7,

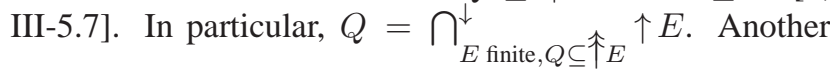
consequence is interpolation: writing $A \ll B$ for $B \subseteq$ $\uparrow A$ (i.e., $A \ll y$ for every $y \in B$ ), if $E \ll x$ in a quasicontinuous dcpo $X$, for some $E \in \operatorname{Fin}(X)$, and $x \in X$, then $E \ll E^{\prime} \ll x$ for some $E^{\prime} \in \operatorname{Fin}(X)$.

Let $\mathcal{Q}(X)$ be the Smyth powerdomain of $X$, i.e., the poset of all non-empty compact saturated subsets $Q$ of $X$, ordered by $\supseteq$. $\mathcal{Q}(X)$ is a continuous depo whenever $X$ is locally compact and sober, with least upper bounds of directed families computed as filtered intersections, and where $Q \ll Q^{\prime}$ iff $Q^{\prime} \subseteq \operatorname{int}(Q)$. Then, the subsets $\square U=\left\{Q^{\prime} \in \mathcal{Q}(X) \mid Q^{\prime} \subseteq U\right\}, U$ open, form a subbase of the topology of $\mathcal{Q}(X)$. Note that $\square$ commutes with finite intersections and directed unions $\left(\square \bigcup_{i \in I}^{\uparrow} U_{i}=\bigcup_{i \in I}^{\uparrow} \square U_{i}\right)$.

If $X$ is a quasi-continuous depo, the above shows that every $Q \in \mathcal{Q}(X)$ can be written as $\bigcap_{E \in \operatorname{Fin}(X), Q \subseteq \uparrow}^{\downarrow} \uparrow_{E}^{\uparrow} E$, i.e., as the directed least upper bound of those non-empty 
finitary compacts $\uparrow E(E \in \operatorname{Fin}(X))$ that are way-below $Q$. In other words, the finitary compacts form a basis of $\mathcal{Q}(X)$.

\section{QRB-Domains}

A deflation on a poset $X$ is a continuous map $f: X \rightarrow$ $X$ such that $f(x) \leq x$ for every $x \in X$, and which takes only finitely many values. An RB-domain is a pointed dcpo with a directed family of deflations $\left(f_{i}\right)_{i \in I}$ such that, for every $x \in X, x=\sup _{i \in I} f_{i}(x)$ [1, Exercise 4.3.11(9)].

We model QRB-domains after RB-domains, replacing single approximating elements $f_{i}(x)$ by finite subsets.

Definition 3.1 (QRB-Domain) $A$ quasi-deflation on $a$ poset $X$ is a continuous map $\varphi: X \rightarrow F i n(X)$ such that $x \in \uparrow \varphi(x)$ for all $x \in X$, and $\operatorname{Im} \varphi$ is finite. We let $\operatorname{Im} \varphi=\bigcup_{x \in X} \varphi(x)$.

A QRB-domain is a pointed dcpo $X$ with a generating family of quasi-deflations, i.e., a directed family of quasideflations $\left(\varphi_{i}\right)_{i \in I}$ with $\uparrow x=\bigcap_{i \in I}^{\downarrow} \uparrow \varphi_{i}(x)$ for each $x \in X$.

We order quasi-deflations pointwise, i.e., $\varphi \leq \psi$ iff $\varphi(x) \leq \sharp \psi(x)$ for every $x \in X$. One can see the finitary compacts $\uparrow \varphi_{i}(x)$ as being smaller and smaller upward closed sets containing $x$.

That $\varphi$ is continuous means that $\varphi$ is monotonic $(x \leq y$ implies $\varphi(x) \leq \sharp \varphi(y)$ ), and that for every directed family $\left(x_{j}\right)_{j \in J}$ of elements of $X, \varphi\left(\sup _{j \in J} x_{j}\right)$ is a finite subset $E$ of $X$ such that $\uparrow E=\bigcap_{i \in I}^{\downarrow} \uparrow \varphi\left(x_{j}\right)$.

It is obvious that every $\mathbf{R B}$-domain is a $\mathbf{Q R B}$-domain.

Lemma 3.2 Let $\varphi$ be a quasi-deflation on $X$. For every $x \in X, \varphi(x) \ll x$.

Proof. Let $\left(x_{j}\right)_{j \in J}$ be a directed family having a least upper bound above $x$. Since $\varphi$ is continuous, $\bigcap_{j \in J}^{\downarrow} \uparrow \varphi\left(x_{j}\right) \subseteq$ $\uparrow \varphi(x)$. But since $\operatorname{Im} \varphi$ is finite, there are only finitely many sets $\varphi\left(x_{j}\right), j \in J$. So $\uparrow \varphi\left(x_{j}\right) \subseteq \uparrow \varphi(x)$ for some $j \in J$. Since $x_{j} \in \uparrow \varphi\left(x_{j}\right), x_{j} \in \uparrow \varphi(x)$, i.e., $\varphi(x) \leq x_{j}$.

Corollary 3.3 Every QRB-domain is quasi-continuous.

In general, QRB-domains are not continuous. E.g., $\mathcal{N}_{2}$ (Figure $2(i i)$ ) is not continuous. However, $\mathcal{N}_{2}$ is a QRB-domain: for all $i, j \in \mathbb{N}$, take $\varphi_{i j}(\omega)=$ $\omega, \varphi_{i j}(0, m)=\{(0, \min (m, i)),(1, j)\}, \varphi_{i j}(1, m)=$ $\{(0, i),(1, \min (m, j))\}$. Then $\left(\varphi_{i j}\right)_{i, j \in \mathbb{N}}$ is the desired directed family of quasi-deflations.

RB-domains are not just continuous domains, they are stably compact, i.e., locally compact, sober, compact and coherent. We say that a topological space is coherent iff the intersection of any two compact saturated subsets is compact (and saturated). In a stably compact space, the intersection of any family of compact saturated subsets is compact. We show that QRB-domains are stably compact as well. For this, we need the following consequence of Rudin's Lemma, a finitary form of well-filteredness:

Proposition 3.4 ([7, III.3-4]) Let $X$ be a dcpo, $\left(E_{i}\right)_{i \in I}$ be a directed family in Fin $(X)$. For every open subset $U$ of $X$, if $\bigcap_{i \in I}^{\downarrow} \uparrow E_{i} \subseteq U$, then $E_{i} \subseteq U$ for some $i \in I$.

Corollary 3.5 Let $X$ be a dcpo. For every open subset $U$ of $X$, the subset $\square_{F i n} U=\{E \in \operatorname{Fin}(X) \mid E \subseteq U\}$ is open in $\operatorname{Fin}(X)$.

Proof. First, $\square_{\text {fin }} U$ is upward closed: if $E \in \square_{\text {fin }} U$ and $E \leq^{\sharp} E^{\prime}$, then $\uparrow E \subseteq U$ (since $U$ is upward-closed) and $\uparrow E^{\prime} \subseteq \uparrow E$, so $\uparrow E^{\prime} \subseteq U$, whence $E^{\prime} \in \square_{\text {fin }} U$. The rest of the claim is Proposition 3.4, verbatim.

Lemma 3.6 Let $X, Y$ be dcpos. For every map $\psi: X \rightarrow$ $\operatorname{Fin}(Y)$, let $\psi^{\dagger}: \operatorname{Fin}(X) \rightarrow \operatorname{Fin}(Y)$ map $E \in \operatorname{Fin}(X)$ to $\bigcup_{x \in E} \psi(x)$ (resp., if $\operatorname{Im} \psi$ is finite, then let $\psi^{*}: \mathcal{Q}(X) \rightarrow$ Fin $(Y)$ map $Q \in \mathcal{Q}(X)$ to $\left.\bigcup_{x \in Q} \psi(x)\right)$. If $\psi$ is continuous, then so is $\psi^{\dagger}\left(\right.$ resp., $\left.\psi^{*}\right)$.

Proof. First, $\psi^{\dagger}$ is monotonic: if $E \leq^{\sharp} E^{\prime}$, i.e., for every $x^{\prime} \in E^{\prime}$, there is an $x \in E$ with $x \leq x^{\prime}$, in particular $\uparrow \psi\left(x^{\prime}\right) \subseteq \uparrow \psi(x)$, then for every $x^{\prime} \in E^{\prime}, \psi\left(x^{\prime}\right) \subseteq$ $\uparrow \psi^{\dagger}(E)$. So $\uparrow \psi^{\dagger}\left(E^{\prime}\right) \subseteq \uparrow \psi^{\dagger}(E)$.

Next, we show continuity. This follows from the claim that $\uparrow \psi^{\dagger}\left(\bigcap_{i \in I}^{\downarrow} \uparrow E_{i}\right)=\bigcap_{i \in I}^{\downarrow} \uparrow \psi^{\dagger}\left(E_{i}\right)$ for every directed family $\left(E_{i}\right)_{i \in I}$ in $\operatorname{Fin}(X)$. The inclusion $\subseteq$ is clear by monotonicity. Conversely, since both sides of the equality are saturated, it is enough to show that every open subset $U$ that contains the left-hand side also contains the righthand side. If $U$ contains $\psi^{\dagger}\left(\bigcap_{i \in I}^{\downarrow} \uparrow E_{i}\right)$, then for every $x \in$ $\bigcap_{i \in I}^{\downarrow} \uparrow E_{i}, \psi(x) \subseteq U$, i.e., $\bigcap_{i \in I}^{\downarrow} \uparrow E_{i} \subseteq \psi^{-1}\left(\square_{\text {fin }} U\right)$. Now $\psi^{-1}\left(\square_{\text {fin }} U\right)$ is open, since $\psi$ is continuous and using Corollary 3.5. By Proposition 3.4, $E_{i} \subseteq \psi^{-1}\left(\square_{\text {fin }} U\right)$ for some $i \in I$, i.e., $\psi^{\dagger}\left(E_{i}\right) \subseteq U$. In particular, $\bigcap_{i \in I}^{\downarrow} \uparrow \psi^{\dagger}\left(E_{i}\right) \subseteq U$, and we conclude.

The arguments are similar for $\psi^{*}$, assuming $\operatorname{Im} \psi$ finite. To show continuity, we need to show that every open subset $U$ that contains $\psi^{*}\left(\bigcap_{i \in I}^{\downarrow} Q_{i}\right)$ also contains $\bigcap_{i \in I}^{\downarrow} \uparrow \psi^{*}\left(Q_{i}\right)$. In this case, $\bigcap_{i \in I}^{\downarrow} Q_{i} \subseteq \psi^{-1}\left(\square_{\mathrm{fin}} U\right)$, from which $Q_{i} \subseteq$ $\psi^{-1}\left(\square_{\text {fin }} U\right)$ for some $i \in I$ by the Hofmann-Mislove Theorem [1, Corollary 7.2.11], which applies since $X$ is sober. Then $U$ contains $\psi^{*}\left(Q_{i}\right)$, and we conclude as above.

One may equate $\operatorname{Fin}(X)$ with the subset of those $Q \in \mathcal{Q}(X)$ that are finitary. Then $\psi^{\dagger}$ is the restriction of $\psi^{*}$ to $\operatorname{Fin}(X)$.

Lemma 3.7 Let $X$ be a QRB-domain, and $\left(\varphi_{i}\right)_{i \in I}$ a generating family of quasi-deflations. For every open subset $U$ of $X, \bigcup_{i \in I}^{\uparrow} \varphi_{i}^{-1}\left(\square_{f i n} U\right)=U$. 
Proof. That the union is directed is because $\varphi_{i}^{-1}\left(\square_{\text {fin }} U\right) \subseteq$ $\varphi_{i^{\prime}}^{-1}\left(\square_{\text {fin }} U\right)$ whenever $\varphi_{i}$ is pointwise below $\varphi_{i^{\prime}}$, i.e., $\varphi_{i}(x) \leq \sharp \varphi_{i^{\prime}}(x)$ for all $x \in X$. Since $x \in \varphi_{i}(x)$ for every $i \in I, \varphi_{i}^{-1}\left(\square_{\text {fin }} U\right) \subseteq U$ : for each $x \in \varphi_{i}^{-1}\left(\square_{\text {fin }} U\right)$, $\varphi_{i}(x)$ contains an element below $x$, and is included in $U$, so $x \in U$, since $U$ is upward closed. Finally, we claim that every element $x$ of $U$ is in $\varphi_{i}^{-1}\left(\square_{\mathrm{fin}} U\right)$ for some $i \in I$. Indeed, $\uparrow x \subseteq U$, so $\bigcap_{i \in I} \uparrow \varphi_{i}(x) \subseteq U$. By Proposition 3.4, $\varphi_{i}(x) \subseteq U$ for some $i \in I$, i.e., $\varphi_{i}(x) \in \square_{\text {fin }} U$.

Lemma 3.8 Let $X$ be a QRB-domain, and $\left(\varphi_{i}\right)_{i \in I}$ a generating family of quasi-deflations. For every compact saturated subset $Q$ of $X, Q=\bigcap_{i \in I}^{\downarrow} \uparrow \varphi_{i}^{*}(Q)$. For every $E \in$ Fin $X, \uparrow E=\bigcap_{i \in I}^{\downarrow} \uparrow \varphi_{i}^{\dagger}(E)$.

Proof. Since $\varphi_{i}(x) \leq x$ for all $x$, and $Q$ is upward-closed, $\uparrow \varphi_{i}^{*}(Q)$ contains $Q$ for every $i \in I$. So $Q \subseteq \bigcap_{i \in I}^{\downarrow} \uparrow \varphi^{*}(Q)$. Conversely, since $Q$ is saturated, it is enough to show that every open $U$ containing $Q$ also contains $\bigcap_{i \in I}^{\downarrow} \uparrow \varphi^{*}(Q)$. Since $Q \subseteq U$, by Lemma 3.7, $Q \subseteq \bigcup_{i \in I} \varphi_{i}^{-1}\left(\square_{\mathrm{fin}} U\right)$. By compactness, $Q \subseteq \varphi_{i}^{-1}\left(\square_{\text {fin }} U\right)$ for some $i \in I$, i.e., for every $x \in Q, \varphi_{i}(x) \subseteq U$. So $\varphi_{i}^{*}(Q) \subseteq U$. The statement about $\varphi^{\dagger}$ is similar.

\section{Theorem 3.9 Every QRB-domain is stably compact.}

Proof. Let $X$ be a QRB-domain, with generating family of quasi-deflations $\left(\varphi_{i}\right)_{i \in I}$. Fix two compact saturated subsets $Q$ and $Q^{\prime}$. We must show that $Q \cap Q^{\prime}$ is compact. Without loss of generality, assume $Q \cap Q^{\prime} \neq \emptyset$. We first claim that there is a directed family $\left(E_{i}\right)_{i \in I}$ in $\operatorname{Fin}(X)$ such that, for all $E, E^{\prime} \in \operatorname{Fin}(X)$ such that $Q \subseteq \uparrow E$ and $Q^{\prime} \subseteq \uparrow E^{\prime}$, $Q \cap Q^{\prime}$ is contained in some $\uparrow E_{i}, i \in I$, with $E_{i} \subseteq \uparrow E \cap \uparrow E^{\prime}$.

Let $E_{i}=\bigcup_{x \in Q \cap Q^{\prime}} \varphi_{i}(x)$ (we cannot write $\varphi_{i}^{*}\left(Q \cap Q^{\prime}\right.$ ), since we do not know yet that $Q \cap Q^{\prime}$ is compact). Since $\uparrow E$ is open and contains $Q$, by Lemma 3.8 and Proposition 3.4 there is an $i \in I$ such that $\uparrow \varphi_{i}^{*}(Q) \subseteq \uparrow E$. Similarly, there is an $i \in I$ such that $\uparrow \varphi_{i}^{*}\left(Q^{\prime}\right) \subseteq \uparrow E^{\prime}$-we take the same $i$, using directedness. Then $E_{i} \subseteq \varphi_{i}^{*}(Q) \cap \varphi_{i}^{*}\left(Q^{\prime}\right) \subseteq \uparrow E \cap$ $\uparrow E^{\prime}$. Let us show that $Q \cap Q^{\prime} \subseteq \uparrow E_{i}$ : for every $x \in Q \cap Q^{\prime}$, $\varphi_{i}(x) \ll x$ by Lemma 3.2; but $\varphi_{i}(x) \subseteq E_{i}$, so $E_{i} \leq \sharp$ $\varphi_{i}(x)$, whence $E_{i} \ll x$.

From this, we deduce that $Q \cap Q^{\prime}=\bigcap_{i \in I}^{\downarrow} \uparrow E_{i}$. (The intersection is filtered, since $\left(E_{i}\right)_{i \in I}$ is directed in $\operatorname{Fin}(X)$.) In one direction, $Q \cap Q^{\prime} \subseteq \uparrow E_{i} \subseteq \uparrow E_{i}$ for each $i \in$ I. Conversely, since $Q \cap Q^{\prime} \neq \emptyset, Q \neq \emptyset$, so $Q=$ $\bigcap_{E \in \operatorname{Fin}(X), Q \subseteq \uparrow}^{\downarrow}{ }_{E}^{\uparrow} E$, hence $\bigcap_{E \in \operatorname{Fin}(X), Q \subseteq \uparrow_{E}}^{\downarrow}{ }^{\uparrow} \subseteq Q$. Similarly, $\bigcap_{E^{\prime} \in \operatorname{Fin}(X), Q^{\prime} \subseteq \uparrow E^{\prime}}^{\downarrow} \uparrow E^{\prime} \subseteq Q^{\prime}$. For all $E, E^{\prime}$ with $Q \subseteq \uparrow E, Q^{\prime} \subseteq \uparrow E^{\prime}$, there is an $i \in I$ such that $\uparrow E_{i} \subseteq \uparrow E \cap$

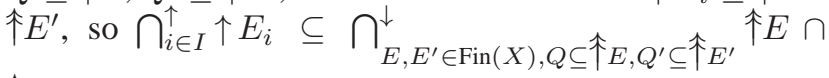
$\uparrow E^{\prime} \subseteq Q \cap Q^{\prime}$.
It follows that $Q \cap Q^{\prime}$ is compact. Explicitly, if $Q \cap Q^{\prime}=$ $\bigcap_{i \in I}^{\downarrow} \uparrow E_{i}$ is contained in a union $U=\bigcup_{j \in J} U_{j}$ of opens, then $E_{i} \in U$ for some $i \in I$ by Proposition 3.4. For each $x \in E_{i}$, pick $j_{x} \in J$ such that $x \in U_{j_{x}}$. Then $Q \cap Q^{\prime} \subseteq$ $\bigcup_{x \in E_{i}} U_{j_{x}}$.

So $X$ is coherent. $X$ is compact since pointed, and also locally compact and sober, as a quasi-continuous dcpo.

The Lawson topology is the smallest topology containing both the Scott-opens and the complements of all sets $\uparrow E, E \in \operatorname{Fin}(X)$. When $X$ is a quasi-continuous dcpo, since $\uparrow E$ is compact saturated and every non-empty compact saturated subset is a filtered intersection of such sets $\uparrow E$, the Lawson topology coincides with the patch topol$o g y$, i.e., the smallest topology containing the original Scott topology and all complements of compact saturated subsets. Every stably compact space is patch-compact, i.e., compact in its patch topology [7, Section VI-6]. So:

\section{Corollary 3.10 Every QRB-domain is Lawson-compact.}

In the sequel, we shall need some form of countability:

Definition 3.11 An $\omega$ QRB-domain is a QRB-domain with a countable generating family of quasi-deflations.

Proposition 3.12 A pointed dcpo $X$ is an $\omega$ QRB-domain iff there is a generating sequence of quasi-deflations $\left(\varphi_{i}\right)_{i \in \mathbb{N}}$, i.e., for every $i, i^{\prime} \in \mathbb{N}, i \leq i^{\prime}, \varphi_{i}(x) \leq^{\sharp} \varphi_{i^{\prime}}(x)$ for every $x \in X$, and $\uparrow x=\bigcap_{i \in \mathbb{N}}^{\downarrow} \uparrow \varphi_{i}(x)$ for every $x \in X$.

Proof. Let $X$ be an $\omega$ QRB-domain, and $\left(\psi_{j}\right)_{j \in \mathbb{N}}$ be a countable generating family of quasi-deflations. Build a sequence $\left(j_{i}\right)_{i \in \mathbb{N}}$ by letting $j_{0}=0$, and $j_{i+1}$ be any $j \in \mathbb{N}$ such that $\psi_{j}$ is above $\psi_{i}$ and $\psi_{j_{i}}$, by directedness. Then let $\varphi_{i}=\psi_{j_{i}}$ for every $i \in \mathbb{N}$. By construction, whenever $i \leq i^{\prime}, \varphi_{i}$ is below $\varphi_{i+1}$. And for every $i \in \mathbb{N}, \psi_{i}$ is below $\varphi_{i}=\psi_{j_{i}}$, so $\uparrow x=\bigcap_{i \in \mathbb{N}}^{\downarrow} \uparrow \varphi_{i}(x)$ for every $x \in X$ Then $\left(\psi_{j_{i}}\right)_{i \in \mathbb{N}}$ is the desired sequence.

Proposition 3.13 A QRB-domain $X$ is an $\omega \mathbf{Q R B}$ domain iff it is countably based, i.e., its topology has a countable subbase.

Proof. Only if: let $\left(\varphi_{i}\right)_{i \in \mathbb{N}}$ be a generating sequence of quasi-deflations on $X$; we claim that the countably many subsets $\uparrow \varphi_{i}(y), y \in \operatorname{Im} \varphi_{i}, i \in \mathbb{N}$, form a subbase of the topology.

It is enough to show that, for every open $U$ and $x \in U$, $x \in \uparrow \varphi_{i}(y)$ for some $y \in \operatorname{Im} \varphi_{i}$ such that $\varphi_{i}(y) \subseteq U$ : since $\uparrow x=\bigcap_{j \in \mathbb{N}}^{\downarrow} \uparrow \varphi_{j}(x) \subseteq U$, use Proposition 3.4 to find $j \in \mathbb{N}$ such that $x \in \uparrow \varphi_{j}(x)$ and $\varphi_{j}(x) \subseteq U$. So there is a $y \in \varphi_{j}(x)$ such that $y \leq x$, and $y \in U$. Repeating the argument on $y$, we find $i \in \mathbb{N}$ such that $y \in \uparrow \varphi_{i}(y)$ and $\varphi_{i}(y) \subseteq U$. Since $y \leq x, x \in \uparrow \varphi_{i}(y)$. 


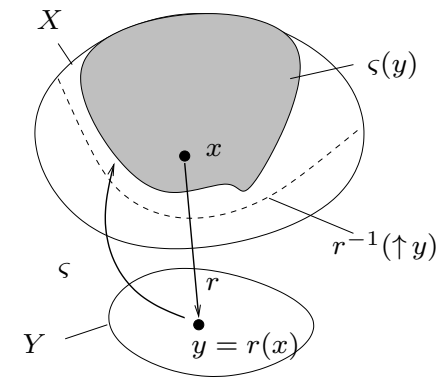

Figure 3. A quasi-retraction

If: let $\left(\varphi_{i}\right)_{i \in I}$ be a generating family of quasi-deflations on $X$, and assume that the topology of $X$ has a countable subbase $\mathcal{B}$. The finite intersections of elements of $\mathcal{B}$ form a base $\mathcal{B}^{\prime}$, i.e., every open is a union of elements of $\mathcal{B}^{\prime}$. Moreover, $\mathcal{B}^{\prime}$ is countable. Write $\mathcal{B}^{\prime}$ as $\left\{U_{k} \mid k \in \mathbb{N}\right\}$, and assume without loss of generality that $U_{k} \neq \emptyset$ for every $k \in \mathbb{N}$. Fix $x_{k} \in U_{k}$ for each $k \in \mathbb{N}$ : by Proposition 3.4 and using directedness, one finds $i_{k} \in I$ such that $\varphi_{i_{k}}\left(x_{k}\right) \subseteq U_{k}$ and $\varphi_{i_{k}}$ is above every $\varphi_{i_{j}}, j \leq k$. Define $\psi_{k}$ as $\varphi_{i_{k}}$. The family $\left(\psi_{k}\right)_{k \in \mathbb{N}}$ is a non-decreasing sequence of deflations, and is generating: $\uparrow x \subseteq \bigcap_{k \in \mathbb{N}} \uparrow \psi_{k}(x)$ since each $\psi_{k}$ is a deflation; conversely, every open containing $x$ contains some $U_{k}$, hence $\uparrow \psi_{k}(x)$, hence $\bigcap_{k \in \mathbb{N}} \uparrow \psi_{k}(x)$.

\section{Quasi-Retracts of Bifinite Domains}

The RB-domains can be characterized as the retracts of the so-called bifinite domains (which we define below). Recall that a retraction of $X$ onto $Y$ is a continuous map $r: X \rightarrow Y$ such that there is continuous map $s: Y \rightarrow X$ (the section) with $r(s(y))=y$ for every $y \in Y$.

We shall show that $(\omega)$ QRB-domains are not just closed under retractions, but under a more relaxed notion that we shall quasi-retractions. In fact, our aim in this section is to show that the pointed $\omega \mathbf{Q R B}$-domains are exactly the quasi-retracts of bifinite domains, up to some details.

For each continuous $r: X \rightarrow Y$, define $\mathcal{Q} r: \mathcal{Q}(X) \rightarrow$ $\mathcal{Q}(Y)$ by $\mathcal{Q} r(Q)=\uparrow\{r(x) \mid x \in Q\}$. $\mathcal{Q} r$ is continuous, since $\mathcal{Q} r^{-1}(\square V)=\square r^{-1}(V)$ for every open $V$.

Definition 4.1 (Quasi-retract) A quasi-retraction $r: X \rightarrow$ $Y$ of $X$ onto $Y$ is a continuous map such that there is a continuous map $\varsigma: Y \rightarrow \mathcal{Q}(X)$ (the quasi-section) such that $\mathcal{Q} r(\varsigma(y))=\uparrow$ y for every $y \in Y$.

The topological space $Y$ is a quasi-retract of $X$ iff there is a quasi-retraction of $X$ onto $Y$.

While a section $s: Y \rightarrow X$ picks an element $s(y)$ in the inverse image $r^{-1}(y)$, continuously, a quasi-section is only required to pick a non-empty compact, saturated collection of elements from $r^{-1}(\uparrow y)$ (see Figure 3 ), continuously again. Every retraction $r$ (with section $s$ ) defines a canonical quasi-retraction: let $\varsigma(y)=\uparrow s(y)$, then $\mathcal{Q} r(\varsigma(y))=$ $\uparrow\{r(z) \mid s(y) \leq z\}=\uparrow r(s(y))=\uparrow y$. The converse fails. Let indeed $\mathbb{N}_{\omega}$ be the dcpo of all natural numbers with an added top element $\omega$, and $X=\mathbb{N}_{\omega}+\mathbb{N}_{\omega}=\{(k, m) \mid k \in$ $\left.\{0,1\}, m \in \mathbb{N}_{\omega}\right\}$, ordered by $(k, m) \leq(\ell, n)$ iff $k=\ell$ and $m \leq n$ (see Figure ?? (iii)). Let $Y=\mathcal{N}_{2}$, and $r$ map $(k, \omega)$ to $\omega$, and $(k, m)$ to itself whenever $m \in \mathbb{N}: r$ is continuous, and has a quasi-section, defined by $\varsigma(k, m)=\uparrow(k, m)$ whenever $m \in \mathbb{N}, \varsigma(\omega)=\{(0, \omega),(1, \omega)\}$. So $r$ is a quasiretraction. But $Y$ is not a retract of $X: X$ is a continuous dcpo, and every retract of a continuous dcpo is again one; recall that $\mathcal{N}_{2}$ is not continuous.

Categorically, as G. Plotkin remarked, in any full subcategory $\boldsymbol{C}$ of $\boldsymbol{T o p}$ where $\mathcal{Q}$ is a monad, every quasi-retraction $r: X \rightarrow Y$ induces a continuous map $\uparrow r\left({ }_{-}\right): X \rightarrow \mathcal{Q}(Y)$, which is then a retraction in the Kleisli category $C_{\mathcal{Q}}$.

Lemma 4.2 Every quasi-retraction $r: X \rightarrow Y$ is onto.

Proof. Let $\varsigma$ be the associated quasi-section. For every $y \in Y, \uparrow y=\mathcal{Q} r(\varsigma(y))$. Since $y \in \mathcal{Q} r(\varsigma(y)), r(x) \leq y$ for some $x \in \varsigma(y)$. But $r(x)$ is then in $\mathcal{Q} r(\varsigma(y))=\uparrow y$, so $y \leq r(x)$. Therefore $y=r(x)$.

The next proposition essentially states that every quasiretract $Y$ of a QRB-domain $X$ is a QRB-domain. However, we need to consider $Y_{\sigma}$ instead, where $Y_{\sigma}$ is the space $Y$ with the Scott topology of its specialization quasiordering $\leq . Y_{\sigma}=Y$ if $Y$ is already a dcpo, but we shall need to consider more general topological spaces later (viz., in Theorem 6.5). $Y$ is a monotone convergence space iff $Y_{\sigma}$ is a dcpo and every open of $Y$ is open in $Y_{\sigma}$ [7].

Proposition 4.3 Let $Y$ be a well-filtered monotone convergence space. If $Y$ is a quasi-retract of an $(\omega) \mathbf{Q R B}$-domain, then $Y_{\sigma}$ is an $(\omega) \mathbf{Q R B}$-domain.

Proof. Let $X$ be a QRB-domain, $r: X \rightarrow Y$ a quasiretraction, and $\varsigma: Y \rightarrow \mathcal{Q}(X)$ a matching quasi-section. Note that $Y$ is pointed: letting $\perp$ the least element of $X$, we claim that $r(\perp)$ is the least element of $Y$. Indeed, for every $y \in Y$, pick some $x \in X$ such that $r(x)=y$ by Lemma 4.2, then $r(\perp) \leq r(x)=y$.

For each quasi-deflation $\varphi$ on $X$, let $\widehat{\varphi}: Y \rightarrow \operatorname{Fin}(Y)$ map $y$ to $\mathcal{Q} r\left(\uparrow \varphi^{*}(\varsigma(y))\right)$. I.e., $\varphi(y)=\uparrow\{r(z) \mid \exists x \in$ $\varsigma(y) \cdot z \in \varphi(x)\}$, where the upward closure $\uparrow$ is taken w.r.t. the specialization quasi-ordering of $Y$ (equivalently, of $Y_{\sigma}$ ).

For every open subset $V$ of $Y, \widehat{\varphi}^{-1}(\square V)$ is the set of all $y \in Y$ such that for every $x \in \varsigma(y)$, for every $z \in \varphi(x), r(z) \in V$. I.e., for every $x \in \varsigma(y)$, $\varphi(x) \subseteq r^{-1}(V)$, that is, $\varsigma(y) \subseteq \varphi^{-1}\left(\square_{\text {fin }} r^{-1}(V)\right)$. So $\widehat{\varphi}^{-1}(\square V)=\varsigma^{-1}\left(\square \varphi^{-1}\left(\square_{\mathrm{fin}} r^{-1}(V)\right)\right)$. Since the latter is open, and the sets $\square V$ form a subbase of the topology of $\mathcal{Q}(X), \widehat{\varphi}$ is continuous from $Y$ to $\operatorname{Fin}(Y)$. 
We need to show that $\widehat{\varphi}$ is Scott-continuous instead. (This would be automatic if $Y$ were a dcpo, but we are not making this assumption.) To this end, observe that $\mathcal{Q} r$ is not only continuous from $\mathcal{Q}(X)$ to $\mathcal{Q}(Y)$, but is also Scott-continuous. Indeed, for every filtered family $\left(Q_{i}\right)_{i \in I}$ of compact saturated subsets of $X, \mathcal{Q} r\left(\bigcap_{i \in I}^{\downarrow} Q_{i}\right) \subseteq$ $\bigcap_{i \in I}^{\downarrow} \mathcal{Q} r\left(Q_{i}\right)$ by monotonicity. For the converse inclusion, every open $U$ containing $\mathcal{Q} r\left(\bigcap_{i \in I}^{\downarrow} Q_{i}\right)$ must be such that $\bigcap_{i \in I}^{\downarrow} Q_{i} \subseteq r^{-1}(U)$, so $Q_{i} \subseteq r^{-1}(U)$ for some $i \in I$ by well-filteredness; so $\mathcal{Q} r\left(\bar{Q}_{i}\right) \subseteq U$, whence $U$ contains $\bigcap_{i \in I}^{\downarrow} \mathcal{Q} r\left(Q_{i}\right)$. Then, for every directed family $\left(y_{i}\right)_{i \in I}$ in $Y, \widehat{\varphi}\left(\sup _{i \in I} y_{i}\right)=\mathcal{Q} r\left(\uparrow \varphi^{*}\left(\bigcap_{i \in I}^{\downarrow} \varsigma\left(y_{i}\right)\right)\right)$ (since $\varsigma: Y \rightarrow \mathcal{Q}(X)$ is continuous and every open of $Y$ is open in $Y_{\sigma}, \varsigma$ is also continuous from $Y_{\sigma}$ to $\mathcal{Q}(X)$, hence Scott-continuous) $=\mathcal{Q} r\left(\bigcap_{i \in I}^{\downarrow} \uparrow \varphi^{*}\left(\varsigma\left(y_{i}\right)\right)\right)$ (Lemma 3.6) $=\bigcap_{i \in I}^{\downarrow} \mathcal{Q} r\left(\uparrow \varphi^{*}\left(\varsigma\left(y_{i}\right)\right)\right)(\mathcal{Q} r$ is Scott-continuous). So $\widehat{\varphi}$ is Scott-continuous from $Y$ to $\operatorname{Fin}(Y)$.

We now claim that $y \in \uparrow \widehat{\varphi}(y)$ for every $y \in Y$. Since $\mathcal{Q} r(\varsigma(y))=\uparrow y, y \in \mathcal{Q} r(\varsigma(y))$, so there is an $x \in \varsigma(y)$ such that $r(x) \leq y$. Now $x \in \uparrow \varphi(x)$, so there is a $z \in \varphi(x)$ such that $z \leq x$. Then $r(z) \leq r(x) \leq y$, so $y \in \widehat{\varphi}(y)$.

Let now $\left(\varphi_{i}\right)_{i \in I}$ be a generating family of quasideflations on $X$. Clearly, if $\varphi_{i}$ is below $\varphi_{j}$, then $\widehat{\varphi}_{i}$ is below $\widehat{\varphi}_{j}$, so $\left(\widehat{\varphi}_{i}\right)_{i \in I}$ is directed.

It remains to show that $\bigcap_{i \in I}^{\downarrow} \uparrow \widehat{\varphi}_{i}(y)=\uparrow y$ for every $y \in Y$. Since $y \in \uparrow \hat{\varphi}(y)$, it remains to show $\bigcap_{i \in I}^{\downarrow} \uparrow \widehat{\varphi}_{i}(y) \subseteq \uparrow y$ : we show that every open $V$ containing $y$ contains $\bigcap_{i \in I}^{\downarrow} \uparrow \widehat{\varphi}_{i}(y)$. Since $y \in V$ and $\mathcal{Q} r(\varsigma(y))=\uparrow y$, $\mathcal{Q} r(\varsigma(y)) \subseteq V$, so $\varsigma(y) \in \mathcal{Q} r^{-1}(\square V)=\square r^{-1}(V)$, i.e., $\varsigma(y) \subseteq r^{-1}(V)$. By Lemma 3.7, $\bigcup_{i \in I}^{\uparrow} \varphi_{i}^{-1}\left(\square_{\mathrm{fin}} r^{-1}(V)\right)=$ $r^{-1}(V)$. Since $\varsigma(y)$ is compact, $\varsigma(y) \subseteq \varphi_{i}^{-1}\left(\square_{\mathrm{fin}} r^{-1}(V)\right)$ for some $i \in I$. So $y$ is in $\varsigma^{-1}\left(\square \varphi_{i}^{-1}\left(\square_{\text {fin }} r^{-1}(V)\right)\right)$, which is equal to $\widehat{\varphi}_{i}^{-1}(\square V)$ (see above). It follows that $V$ contains $\widehat{\varphi}_{i}(y)$, hence $\bigcap_{i \in I}^{\downarrow} \uparrow \widehat{\varphi}_{i}(y)$. So $Y_{\sigma}$ is a QRB-domain.

The case of $\omega \mathbf{Q R B}$-domains is similar, where now $\left(\varphi_{i}\right)_{i \in \mathbb{N}}$ is a generating sequence of quasi-deflations.

The assumption on $Y$ is always satisfied in the following important case. A quasi-retraction $r: X \rightarrow Y$, with quasisection $\varsigma: Y \rightarrow \mathcal{Q}(X)$, is a quasi-projection iff, additionally, $x \in \varsigma(r(x))$ for every $x \in X$. In this case, we say that $Y$ is a quasi-projection of $X$. The quasi-retraction of Figure 3 is meant to be a quasi-projection: $x$ is in the gray area $\varsigma(y)$. The following is reminiscent of the fact that every retract of a stably compact space is again stably compact [19, Proposition, bottom of p.153, and subsequent discussion]; see Appendix A for a proof.

Proposition 4.4 Every $T_{0}$ quasi-projection $Y$ of a stably compact space $X$ is stably compact.

Let us turn to bifinite domains. A pointed dcpo $X$ is a bifinite domain iff there is a non-decreasing sequence of idempotent deflations $\left(f_{i}\right)_{i \in \mathbb{N}}$ such that, for every $x \in X$, $x=\sup _{i \in \mathbb{N}} f_{i}(x)$ [1, Theorem 4.2.6]. By idempotent, we mean that $f_{i} \circ f_{i}=f_{i}$. An alternative definition is as follows. First, a retraction $p: X \rightarrow Y$, with section $e: Y \rightarrow X$, is a projection iff, additionally, $e(p(x)) \leq x$ for every $x \in X$; then $e$ is usually called an embedding; $e$ is left-adjoint to $p$, hence each of $e, p$ is determined uniquely by the other one. An expanding sequence of dcpos is a family $\left(X_{i}\right)_{i \in \mathbb{N}}$ with projection maps $\left(p_{i j}\right)_{i, j \in \mathbb{N}, i \leq j}$ where $p_{i j}: X_{j} \rightarrow X_{i}, p_{i i}=\operatorname{id}_{X_{i}}$, and $p_{i k}=p_{i j} \circ p_{j k}$ whenever $i \leq j \leq k$ [1, Definition 3.3.6]. Given any $\vec{x} \in \prod_{i \in \mathbb{N}} X_{i}$,

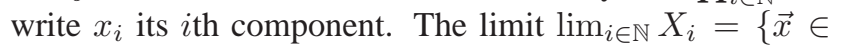
$\left.\prod_{i \in \mathbb{N}} X_{i} \mid \forall i \leq j \in \mathbb{N} \cdot p_{i j}\left(x_{j}\right)=x_{i}\right\}$ is a depo, and is both a limit and a colimit in suitable categories (this is a bilimit). Then the bifinite domains are (up to isomorphism) the bilimits of expanding systems of finite, pointed posets [1, Theorem 4.2.7].

The key lemma to prove Theorem 4.6 below is the following. It is tempting to think that this lemma is merely Rudin's Lemma [7, III-3.3], itself a key lemma in the theory of quasi-continuous depos. But this is wrong. Rudin's Lemma would only secure the existence of a directed family $Z$ whose least upper bound is $y$, and which intersects each $\varphi_{i}(y)$; but $Z$ may intersect each $\varphi_{i}(y)$ in more than one element $y_{i}$.

Lemma 4.5 Let $Y$ be a dcpo, $y \in Y$, and $\left(E_{i}^{0}\right)_{i \in \mathbb{N}}$ a nondecreasing sequence in $\operatorname{Fin}(Y)$ (w.r.t. $\left.\leq^{\sharp}\right)$ such that $\uparrow y=$ $\bigcap_{i \in \mathbb{N}}^{\downarrow} E_{i}^{0}$. There is a non-decreasing sequence $\left(y_{i}\right)_{i \in \mathbb{N}}$ in $Y$ such that $y_{i} \in E_{i}^{0}$ for every $i \in \mathbb{N}$, and $\sup _{i \in \mathbb{N}} y_{i}=y$.

Proof. Consider the collection $\mathcal{D}$ of all sequences $\left(E_{i}\right)_{i \in \mathbb{N}}$ of non-empty sets $E_{i} \subseteq E_{i}^{0}$ such that $y \in \uparrow E_{i}$ and $E_{i} \leq \sharp$ $E_{i+1}$ for every $i \in \mathbb{N}$. $\mathcal{D}$ is non-empty, since $\left(E_{i}^{0}\right)_{i \in I}$ is in it. Order $\mathcal{D}$ by pointwise inclusion. By Zorn's Lemma, $\mathcal{D}$ has a minimal element $\left(E_{i}\right)_{i \in \mathbb{N}}$. Assume for the sake of contradiction that some $E_{j}$ contains at least two elements, and pick $j$ minimal. Define $y_{i}$ for every $i \leq j$ as follows: $y_{j}$ is any element of $E_{j}$ below $y$ (using $y \in \uparrow E_{j}$ ), and, by induction on $j-i, y_{i-1}$ is any element of $E_{i-1}$ below $y_{i}$ (using $E_{i-1} \leq \sharp E_{i}$ ). The family $\left(E_{i}^{\prime}\right)_{i \in \mathbb{N}}$ defined by $E_{i}^{\prime}=$ $E_{i}$ for every $i \geq j+1$, and $E_{i}^{\prime}=\left\{y_{i}\right\}$ for each $i \leq j$ is then in $\mathcal{D}$, and contradicts the minimality of $\left(E_{i}\right)_{i \in \mathbb{N}}$. So each $E_{i}$ contains exactly one element, say $y_{i}$; for each $i \in \mathbb{N}$, $y_{i} \in E_{i}^{0}, y_{i} \leq y$, and $\left(y_{i}\right)_{i \in \mathbb{N}}$ is non-decreasing.

In particular, $\sup _{i \in \mathbb{N}} y_{i} \in \bigcap_{i \in \mathbb{N}}^{\downarrow} \uparrow E_{i}^{0}=\uparrow y$. So $y \leq$ $\sup _{i \in \mathbb{N}} y_{i}$. Since $y_{i} \leq y$ for each $i$, equality follows.

Theorem 4.6 The following are equivalent for a dcpo $Y$ :

(i) $Y$ is an $\omega \mathbf{Q R B}-d o m a i n$;

(ii) $Y$ is a well-filtered quasi-retract of a bifinite domain;

(iii) $Y$ is a quasi-projection of a bifinite domain. 
Proof. $\quad$ (iii) $\Rightarrow($ ii $) . Y$ is stably compact by Proposition 4.4 since every bifinite domain is stably compact. So $Y$ is wellfiltered [1, Corollary 7.2.11].

(ii) $\Rightarrow(i)$. Let $Y$ be well-filtered. As a dcpo, it is a monotone convergence space. Write $Y$ as a quasiretract of a bifinite domain $X . X$ is an RB-domain, hence an $\omega$ QRB-domain. By Proposition 4.3, $Y=Y_{\sigma}$ is an $\omega$ QRB-domain.

$(i) \Rightarrow($ iii $)$. Let $Y$ be an $\omega$ QRB-domain, with generating sequence of quasi-deflations $\left(\varphi_{i}\right)_{i \in \mathbb{N}^{*}}$. Let $X$ be the set of all non-decreasing sequences $\vec{y}=\left(y_{i}\right)_{i \in \mathbb{N}}$ in $Y$ such that $y_{i} \in \bigcup_{j \leq i} \operatorname{Im} \varphi_{j}$, and $y_{i} \in \uparrow \varphi_{i}\left(\sup _{k \in \mathbb{N}} y_{k}\right)$. Order $X$ componentwise. As in [14, Theorem 4.9, Theorem 4.1], $X$ is a bifinite domain: for each $i_{0} \in \mathbb{N}$, consider the idempotent deflation $f_{i_{0}}$ defined by $f_{i_{0}}(\vec{y})=$ $\left(y_{\min \left(i, i_{0}\right)}\right)_{i \in \mathbb{N}}$. To show that this is well-defined, we must show that $y_{\min \left(i, i_{0}\right)} \in \uparrow \varphi_{i}\left(\sup _{k \in \mathbb{N}} y_{\min \left(k, i_{0}\right)}\right)$, i.e., that $y_{\min \left(i, i_{0}\right)} \in \uparrow \varphi_{i}\left(y_{i_{0}}\right)$. If $i \leq i_{0}$, then $y_{\min \left(i, i_{0}\right)}=y_{i} \in$ $\uparrow \varphi_{i}\left(\sup _{k \in \mathbb{N}} y_{k}\right) \subseteq \uparrow \varphi_{i}\left(y_{i_{0}}\right)$ since $\vec{y} \in X$ and $\varphi_{i}$ is monotonic, else $y_{\min \left(i, i_{0}\right)}=y_{i_{0}} \in \uparrow \varphi_{i}\left(y_{0}\right)$ since $\varphi_{i}$ is a quasideflation. It is easy to see that $f_{i_{0}}$ is Scott-continuous.

Let now $r: X \rightarrow Y$ map $\vec{y}$ to $\sup _{i \in \mathbb{N}} y_{i}$, and $\varsigma$ map $y \in Y$ to $\left\{\vec{y} \in X \mid \forall i \in \mathbb{N} \cdot y_{i} \in \uparrow \varphi_{i}(y)\right\}: \varsigma(y)$ is nonempty, using Lemma 4.5.

Next, we claim that $\varsigma(y)$ is compact in $X$. For each $i_{0} \in \mathbb{N}$, let $Q_{i_{0}}=\left\{\vec{y} \in X \mid \forall i \leq i_{0} \cdot y_{i} \in \uparrow \varphi_{i}(y)\right\}$. Let $K_{i_{0}}$ be the set of all elements $\vec{y}$ of $Q_{i_{0}}$ such that $y_{i}=y_{i_{0}}$ for every $i \geq i_{0}$. Note that $K_{i_{0}}$ is finite, (recall that each $y_{i}$ with $i \leq i_{0}$ is taken from the finite set $\bigcup_{j \leq i} \operatorname{Im} \varphi_{j}$ ), and that $Q_{i_{0}}=\uparrow K_{i_{0}}$. Indeed, for every $\vec{y} \in \bar{Q}_{i_{0}}$, its image $f_{i_{0}}(\vec{y})$ by the idempotent deflation $f_{i_{0}}$ is in $K_{i_{0}}$, and is below $\vec{y}$. So $Q_{i_{0}}$ is (finitary) compact. Every bifinite domain is stably compact [1, Theorem 4.2.18], and any intersection of saturated compacts in a stably compact space is (saturated) compact, so $\varsigma(y)=\bigcap_{i_{0} \in \mathbb{N}} Q_{i_{0}}$ is saturated compact. So $\varsigma(y) \in \mathcal{Q}(Y)$. It is easy to see that $\varsigma$ and $r$ are Scottcontinuous.

Given any $z \in \mathcal{Q} r(\varsigma(y))$, there is a non-decreasing sequence $\vec{y}$ in $\varsigma(y)$, i.e., such that $y_{i} \in \uparrow \varphi_{i}(y)$ for every $i \in \mathbb{N}$, with $\sup _{i \in \mathbb{N}} y_{i} \leq z$. Since $y_{i} \in \uparrow \varphi_{i}(y)$ for every $i \in \mathbb{N}, \sup _{i \in \mathbb{N}} y_{i}$ is in $\bigcap_{i \in \mathbb{N}} \uparrow \varphi_{i}(y)=\uparrow y$, so $z \in \uparrow y$. That is, $\mathcal{Q} r(\varsigma(y)) \subseteq \uparrow y$. Conversely, we note that $y \in \mathcal{Q} r(\varsigma(y))$, i.e., that there is a sequence $\vec{y}$ with $y_{i} \in \uparrow \varphi_{i}(y)$ for every $i \in \mathbb{N}$, and such that $\sup _{i \in \mathbb{N}} y_{i} \leq y$ : this is by Lemma 4.5. So $\mathcal{Q} r(\varsigma(y))=\uparrow y$. Finally, for every $\vec{y} \in X$, since $y_{i} \in \uparrow \varphi_{i}\left(\sup _{k \in \mathbb{N}} y_{k}\right)=\uparrow \varphi_{i}(r(\vec{y}))$ for every $i \in \mathbb{N}$, $\vec{y} \in \varsigma(r(\vec{y}))$. So $r$ is a quasi-projection.

\section{Products, Bilimits}

If $\left(\varphi_{i}\right)_{i \in X}$ (resp. $\left.\left(\psi_{j}\right)_{j \in J}\right)$ is a generating family of quasi-deflations on $X$ (resp. $Y$ ), $\left(\chi_{i j}\right)_{i \in I, j \in J}$ is one on $X \times Y$, where $\chi_{i j}(x, y)=\varphi_{i}(x) \times \psi_{j}(y)($ see Appendix B), so:
Lemma 5.1 For any two ( $\omega)$ QRB-domains $X, Y, X \times Y$, with the product ordering, is an $(\omega) \mathbf{Q R B}$-domain.

Bilimits are harder to deal with. But the difficulty was solved by Jung [14, Section 4.1] in the case of RB-domains and deflations, and we proceed in a very similar way. All proofs are in Appendix C.

Consider any set $G$ of functions $\psi$ from $X$ to $\operatorname{Fin}(X)$ such that $\psi(x) \leq \sharp\{x\}$, i.e., $x \in \uparrow \psi(x)$, for every $x \in$ $X$. We say that $G$ is $q f$ s (for quasi-finitely separating) iff given any finitely many pairs $\left(E_{k}, x_{k}\right) \in \operatorname{Fin}(X) \times X$ with $E_{k} \ll x_{k}, 1 \leq k \leq n$, there is a $\psi \in G$ that separates the pairs, i.e., such that $E_{k} \leq \sharp \psi\left(x_{k}\right) \leq \sharp\left\{x_{k}\right\}$ (equivalently, $\left.x_{k} \in \uparrow \psi\left(x_{k}\right) \subseteq \uparrow E_{k}\right)$ for every $k, 1 \leq k \leq n$.

Proposition 5.2 Let $X$ be a poset. Then $X$ is a QRBdomain iff $X$ is a quasi-continuous dcpo and the set $G$ of quasi-deflations on $X$ is qfs.

Proof. (Sketch.) This is a variant on [14, Theorem 4.5], and is proved similarly. In the if direction, assume $G$ qfs, and define $H=\left\{\varphi^{\dagger} \circ \varphi \mid \varphi \in G\right\}$. It is easy to see that $H$ is a generating family of quasi-deflations provided it is directed. To show that it is directed, pick $\varphi$ and $\varphi^{\prime}$ from $G$, let $E=\operatorname{Im} \varphi, E^{\prime}=\operatorname{Im} \varphi^{\prime}$. Using the form of interpolation available in quasi-continuous dcpos, find a finite set $E_{y}$ such that $\varphi(y) \ll E_{y} \ll y$ for each $y \in E$, and similarly $E_{y^{\prime}}^{\prime}$ such that $\varphi^{\prime}\left(y^{\prime}\right) \ll E_{y^{\prime}}^{\prime} \ll y^{\prime}$ for each $y^{\prime} \in E^{\prime}$. By qfs, find $\psi \in G$ that separates the pairs $\left(E_{y}, y\right),(\varphi(y), z),\left(E_{y^{\prime}}^{\prime}, y^{\prime}\right)$, and $\left(\varphi^{\prime}\left(y^{\prime}\right), z^{\prime}\right)$ for all $y \in E, z \in E_{y}, y^{\prime} \in E^{\prime}, z^{\prime} \in E_{y^{\prime}}^{\prime}$. One checks that $\varphi^{\dagger} \circ \varphi$ and $\varphi^{\prime \dagger} \circ \varphi^{\prime}$ are below $\psi^{\dagger} \circ \psi$.

Theorem 5.3 Any bilimit of $\omega \mathbf{Q R B}$-domains is an $\omega$ QRB-domain.

Proof. (Sketch.) As in [14, Theorem 4.5]. Let $\left(X_{i}\right)_{i \in \mathbb{N}}$ be an expanding sequence of QRB-domains, $X$ its bilimit, $p_{i}: X \rightarrow X_{i}$ the canonical projection and $e_{i}: X_{i} \rightarrow X$ the canonical embedding. One checks that, for any finite set of pairs $\left(\vec{E}_{k}, \vec{x}_{k}\right)$ with $\vec{E}_{k} \ll \vec{x}_{k}$ in $X$, one can separate them by quasi-deflations of the form Fin $e_{i} \circ \varphi \circ p_{i}$, where $i \in \mathbb{N}$, $\varphi$ is a quasi-deflation on $X_{i}$, and Fin $e_{i}(E)=\left\{e_{i}(x) \mid x \in\right.$ $E\}$; then use Proposition 5.2.

\section{The Probabilistic Powerdomain}

Let $X$ be a fixed topological space, and let $\mathcal{O}(X)$ be the lattice of open subsets of $X$. A continuous valuation $\nu$ on $X$ [13] is a map from $\mathcal{O}(X)$ to $\mathbb{R}^{+}$such that $\nu(\emptyset)=0$, which is monotonic $(\nu(U) \leq \nu(V)$ whenever $U \subseteq V)$, modular $(\nu(U \cup V)+\nu(\mathcal{U} \cap V)=\nu(U)+\nu(V)$ for all opens $U, V)$, and continuous $\left(\nu\left(\bigcup_{i \in I}^{\uparrow} U_{i}\right)=\sup _{i \in I} \nu\left(U_{i}\right)\right.$ for every directed family $\left(U_{i}\right)_{i \in I}$ of opens). A (sub)probability valuation $\nu$ is additionally such that $\nu$ is (sub)normalized, 
i.e., that $\nu(X)=1(\nu(X) \leq 1)$. Let $\mathbf{V}_{1}(X)\left(\mathbf{V}_{\leq 1}(X)\right)$ be the dcpo of all (sub)probability valuations on $X$, ordered pointwise, i.e., $\nu \leq \nu^{\prime}$ iff $\nu(U) \leq \nu^{\prime}(U)$ for every open $U . \mathbf{V}_{1}\left(\mathbf{V}_{\leq 1}\right)$ defines a endofunctor on the category of dcpos, and its action is defined on morphisms $f$ by $\mathbf{V}_{1} f(\nu)(U)=\nu\left(f^{-1}(U)\right)$.

If $Y$ is a retract of $X$, then $\mathbf{V}_{1}(Y)$ is easily seen to be a retract of $\mathbf{V}_{1}(X)$, using the $\mathbf{V}_{1}$ endofunctor. The following result is more involved. Here we need to replace the Scott topology on $\mathbf{V}_{1}(X)$ by the weak topology, which is the smallest one containing the subbasic opens $[U>r]$, defined as $\left\{\nu \in \mathbf{V}_{1}(X) \mid \nu(U)>r\right\}$, for each open subset $U$ of $X$ and $r \in \mathbb{R}$. When $X$ is a continuous pointed dcpo, the Kirch-Tix Theorem states that it coincides with the Scott topology (see [2], who attribute it to Tix [21, Satz 4.10], who in turn attributes it to Kirch [18, Satz 8.6]). However, the weak topology is better behaved in the general case. Let $\mathbf{V}_{1 w k}(X)$ be $\mathbf{V}_{1}(X)$ with its weak topology.

Theorem 6.1 (Key Claim) If $Y$ is a quasi-projection of $X$, and $X$ is stably compact, then $\mathbf{V}_{1 w k}(Y)$ is a quasiprojection of $\mathbf{V}_{1 w k}(X)$.

Proof. Let $r: X \rightarrow Y$ be the quasi-retraction, $\varsigma: Y \rightarrow$ $\mathcal{Q}(X)$ be the quasi-section. Define $r^{\prime}: \mathbf{V}_{1 w k}(X) \rightarrow$ $\mathbf{V}_{1 w k}(Y)$ by $r^{\prime}=\mathbf{V}_{1} r$. To define $\varsigma^{\prime}: \mathbf{V}_{1 w k}(Y) \rightarrow$ $\mathcal{Q}\left(\mathbf{V}_{1 w k}(X)\right)$, we require some auxiliary machinery.

Let $\mathbb{R}_{\sigma}^{+}$be $\mathbb{R}^{+}$with its Scott topology, and $\left\langle X \rightarrow \mathbb{R}_{\sigma}^{+}\right\rangle$ be the poset of all bounded continuous maps from $X$ to $\mathbb{R}_{\sigma}^{+}$. $\quad \mathbf{V}_{1 w k}(X)$ is canonically isomorphic to the space $\mathbf{P}_{1 w k}^{\triangle}(X)$ of so-called continuous normalized linear previsions on $X$ [10]. A prevision $F$ on $X$ is a map $F$ : $\left\langle X \rightarrow \mathbb{R}_{\sigma}^{+}\right\rangle \rightarrow \mathbb{R}_{\sigma}^{+}$such that $F(a f)=a F(f)$ for every $a \in \mathbb{R}^{+}$. A prevision $F$ is lower, or superlinear, iff $F\left(h+h^{\prime}\right) \geq F(h)+F\left(h^{\prime}\right)$ for every $h, h^{\prime}$, upper, or sublinear, iff $F\left(h+h^{\prime}\right) \leq F(h)+F\left(h^{\prime}\right)$ for every $h, h^{\prime}$, linear iff $F\left(h+h^{\prime}\right)=F(h)+F\left(h^{\prime}\right)$, normalized iff $F(a+h)=a+F(h)$ for all $h \in\left\langle X \rightarrow \mathbb{R}_{\sigma}^{+}\right\rangle$and $a \in \mathbb{R}^{+}$ [10]. On any space of previsions $Y$, the weak topology is the smallest such that $[h>r]=\{F \in Y \mid F(h)>r\}$ is open for every $h \in\left\langle X \rightarrow \mathbb{R}_{\sigma}^{+}\right\rangle$and $r \in \mathbb{R}$. Let $\mathbf{P}_{1 w k}^{\triangle}(Y)$, resp. $\nabla \mathbf{P}_{1 w k}(X)$, be the space of continuous normalized linear (resp. lower) previsions on $X$. The homeomorphism $\mathbf{V}_{1 w k}(Y) \cong \mathbf{P}_{1 w k}^{\triangle}(Y)$ maps $\nu \in \mathbf{V}_{1 w k}(X)$ to the prevision $\lambda h \in\left\langle X \rightarrow \mathbb{R}_{\sigma}^{+}\right\rangle \cdot \oint_{x \in X} h(x) d \nu$, where $\oint$ denotes Choquet integral [9], and conversely every $G \in \mathbf{P}_{1 w k}^{\triangle}(X)$ to $U \in \mathcal{O}(X) \mapsto G\left(\chi_{U}\right)$, where $\chi_{U}$ is the characteristic function of $U$. Note that $r^{\prime}=\mathbf{V}_{1} r$ transports through this isomorphism to the map sending each $G^{\prime} \in \mathbf{P}_{1 w k}^{\triangle}(X)$ to $\lambda h^{\prime} \in\left\langle Y \rightarrow \mathbb{R}_{\sigma}^{+}\right\rangle \cdot G^{\prime}\left(h^{\prime} \circ r\right) \in \mathbf{P}_{1 w k}^{\triangle}(Y)$.

Given $h \in\left\langle X \rightarrow \mathbb{R}_{\sigma}^{+}\right\rangle$, let $h_{*}$ map each $Q \in \mathcal{Q}(X)$ to $\min _{x \in Q} h(x)$. Then $h_{*}$ is in $\left\langle\mathcal{Q}(X) \rightarrow \mathbb{R}_{\sigma}^{+}\right\rangle$(see [9]). We claim that:
(i) $h_{*} \circ i \circ r \leq h$
(ii) $h^{\prime} \leq\left(h^{\prime} \circ r\right)_{*} \circ i$

for all $h \in\left\langle X \rightarrow \mathbb{R}_{\sigma}^{+}\right\rangle, h^{\prime} \in\left\langle Y \rightarrow \mathbb{R}_{\sigma}^{+}\right\rangle$. For $(i)$, for every $x \in X, h_{*} \circ i \circ r(x)=\min _{z \in i(r(x))} h(z) \leq h(x)$, since $x \in i(r(x))$. (This is where we use the fact that $r$ is a quasi-projection.) For (ii), for every $y \in Y$, for every $x \in i(y), r(x)$ is in $\mathcal{Q} r(i(y))=\uparrow y$, so $y \leq r(x)$. So $\left(h^{\prime} \circ r\right)_{*} \circ i(y)=\min _{x \in i(y)} h^{\prime}(r(x)) \geq h^{\prime}(y)$. (We let the reader check that, in fact, $\left(h^{\prime} \circ r\right)_{*} \circ i=h^{\prime}$.)

We also note that the mapping $h \mapsto h_{*}$ is Scott-continuous: by [9, Proposition 1], $h_{*}(Q)=$ $\oint_{x \in X} h(x) d \mathfrak{u}_{Q}$, where the unanimity game $\mathfrak{u}_{Q}$ is continuous for every compact saturated set $Q$ [9, Section 3], and observe that Choquet integration along continuous games is Scott-continuous in the integrated function $h$ [9, Section 4].

We recall from [10, Proposition 4] that there is a map CCoeur $1: \nabla \mathbf{P}_{1 w k}(X) \rightarrow \mathcal{Q}\left(\mathbf{P}_{1 w k}^{\triangle}(X)\right)$ sending each continuous normalized lower prevision $F$ to its heart $C$ Coeur $_{1}(F)=\left\{G \in \mathbf{P}_{1 w k}^{\triangle}(X) \mid F \leq G\right\}$. When $X$ is stably compact, $C C$ oeur $1(F)$ is always non-empty, compact, and saturated. (The first two properties are nontrivial.) Moreover, $C C_{\text {oeur }}$ is continuous.

Equate $\mathbf{V}_{1 w k}(X)$ with $\mathbf{P}_{1 w k}^{\triangle}(X)$ : from now on, probability valuations will be taken to be elements $G$ of $\mathbf{P}_{1 w k}^{\triangle}(X)$. Define $\varsigma^{\prime}: \mathbf{V}_{1 w k}(Y) \rightarrow \mathcal{Q}\left(\mathbf{V}_{1 w k}(X)\right)$ by $\varsigma^{\prime}(G)=C_{C o e u r}\left(F_{G}^{-}\right)$, where $F_{G}^{-}(h)=G\left(h_{*} \circ i\right)$. One checks easily that $F_{G}^{-}$is a lower normalized prevision. It is continuous because $G$ is, and because $h \mapsto h_{*}$ is Scottcontinuous. So $\varsigma^{\prime}(G)$ is well-defined.

The map sending $G$ to $F_{G}^{-}$is also continuous, since the inverse image of $[h>r]$ is $\left[h_{*} \circ i>r\right]$. Since $\varsigma^{\prime}$ is the composition of $C$ Coeur $_{1}$ with this map, it is also continuous.

Let us show that $\mathcal{Q} r^{\prime}\left(\varsigma^{\prime}(G)\right)=\uparrow G$ for every $G \in$ $\mathbf{P}_{1 w k}^{\triangle}(Y)$. We first use Keimel's topological version of Roth's Sandwich Theorem [17, Theorem 8.2]: in a topological cone $C$ (i.e., a space $C$ with a continuous addition $+: C \times C \rightarrow C$ and a continuous scalar multiplication . : $\mathbb{R}_{\sigma}^{+} \times C \rightarrow C$ satisfying the expected equalities), for every superlinear continuous map $q: C \rightarrow \overline{\mathbb{R}_{\sigma}^{+}}$(where $\overline{\mathbb{R}_{\sigma}^{+}}$ is $\mathbb{R} \cup\{+\infty\}$ with its Scott topology), for every sublinear map $p: C \rightarrow \overline{\mathbb{R}_{\sigma}^{+}}$such that $q \leq p$, there is a continuous linear map $\Lambda: C \rightarrow \overline{\mathbb{R}_{\sigma}^{+}}$such that $q \leq \Lambda \leq p$. Let $C=\left\langle X \rightarrow \mathbb{R}_{\sigma}^{+}\right\rangle$. This is a continuous poset as soon as $X$ is locally compact; for our purposes, we may use [11, Proposition 7.11], which states this, and a bit more, in the case where $X$ is stably compact. Also addition and scalar multiplication are Scott-continuous. Since the Scott topology of the product of two continuous posets is the product of the Scott topologies, $C$ is a continuous cone.

Let $q=F_{G}^{-}$, i.e., $q(h)=G\left(h_{*} \circ i\right), p(h)=$ $\sup _{h^{\prime} \in\left\langle Y \rightarrow \mathbb{R}_{\sigma}^{+}\right\rangle, h^{\prime} \text { or } \leq h} G\left(h^{\prime}\right)$. Since $F_{G}^{-}$is a continuous lower prevision, $q$ is superlinear and continuous. It is easy to see that $p$ is sublinear. Moreover, $q \leq p$ : for each 


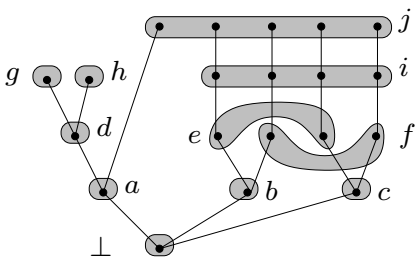

Figure 4. The path space of Figure $2(i)$

$h$, let $h^{\prime}=h_{*} \circ i$, then $h^{\prime} \circ r \leq h$ by $(i)$ above, so that $q(h)=G\left(h^{\prime}\right) \leq p(h)$. Find a continuous linear map $\Lambda: C \rightarrow \overline{\mathbb{R}_{\sigma}^{+}}$such that $q=F_{G}^{-} \leq \Lambda \leq p$ by Keimel's Theorem. Since $r$ is onto (Lemma 4.2), whenever $h^{\prime} \circ r \leq h, \sup _{y \in Y} h^{\prime}(y) \leq \sup _{x \in X} h(x)$; since $G$ is normalized, $p(h) \leq \sup _{x \in X} h(x)<+\infty$. In particular, $\Lambda$ is in fact a continuous linear prevision. $\Lambda$ is also normalized: for every constant $a \in \mathbb{R}^{+}, a=q(a) \leq \Lambda(a) \leq p(a) \leq a$, so $\Lambda(a+h)=\Lambda(a)+\Lambda(h)=a+\Lambda(h)$.

Recall that $\varsigma^{\prime}(G)=C$ Coeur $_{1}\left(F_{G}^{-}\right)$. The above allows us to show that $G$ is in $\mathcal{Q} r^{\prime}\left(\varsigma^{\prime}(G)\right)$, i.e., that there is a $G^{\prime} \in$ $\varsigma^{\prime}(G)$ (i.e., in $\mathbf{P}_{1 w k}^{\triangle}(X)$ and such that $F_{G}^{-} \leq G^{\prime}$ ) such that $r^{\prime}\left(G^{\prime}\right) \leq G$. Take $G^{\prime}=\Lambda$ : since $q=F_{G}^{-} \leq \Lambda \leq p$, we obtain $F_{G}^{-} \leq G^{\prime}$, and on the other hand $G^{\prime}\left(h^{\prime} \circ r\right)=$ $\Lambda\left(h^{\prime} \circ r\right) \leq p\left(h^{\prime} \circ r\right)=\sup _{h^{\prime \prime} \in\left\langle Y \rightarrow \mathbb{R}_{\sigma}^{+}\right\rangle, h^{\prime \prime} \circ r \leq h^{\prime} \circ r} G\left(h^{\prime \prime}\right)$. Since $r$ is onto, $h^{\prime \prime} \circ r \leq h^{\prime} \circ r$ entails $h^{\prime \prime} \leq h^{\prime}$, hence $G\left(h^{\prime \prime}\right) \leq G\left(h^{\prime}\right)$. So $G^{\prime}\left(h^{\prime} \circ r\right) \leq G\left(h^{\prime}\right)$.

Conversely, we claim that $\mathcal{Q} r^{\prime}\left(\varsigma^{\prime}(G)\right)$ is included in $\uparrow G$. This is easier: every element $\Lambda$ of $\mathcal{Q} r^{\prime}\left(\varsigma^{\prime}(G)\right)$ is above some $r^{\prime}\left(G^{\prime}\right)$ with $G^{\prime} \in \varsigma^{\prime}(G)$ (so that $\left.F_{G}^{-} \leq G^{\prime}\right)$. Then $\Lambda\left(h^{\prime}\right) \geq$ $r^{\prime}\left(G^{\prime}\right)\left(h^{\prime}\right)=G^{\prime}\left(h^{\prime} \circ r\right) \geq F_{G}^{-}\left(h^{\prime} \circ r\right)=G\left(\left(h^{\prime} \circ r\right)_{*} \circ i\right) \geq$ $G\left(h^{\prime}\right)$ by $(i i)$, for every $h^{\prime} \in\left\langle Y \rightarrow \mathbb{R}_{\sigma}^{+}\right\rangle$. So $\Lambda \in \uparrow G$.

So $\mathcal{Q} r^{\prime}\left(\varsigma^{\prime}(G)\right)=\uparrow G$ for every $G \in \mathbf{P}_{1 w k}^{\triangle}(Y)$, hence $\mathbf{V}_{1 w k}(Y)$ is a quasi-retract of $\mathbf{V}_{1 w k}(X)$.

Finally, we show that $G^{\prime} \in \varsigma^{\prime}\left(r^{\prime}\left(G^{\prime}\right)\right)$ for every $G^{\prime} \in$ $\mathbf{P}_{1 w k}^{\triangle}(X)$. By $(i)$, for every $h \in\left\langle X \rightarrow \mathbb{R}_{\sigma}^{+}\right\rangle, G^{\prime}\left(h_{*} \circ i \circ\right.$ $r) \leq G^{\prime}(h)$, i.e., $r^{\prime}\left(G^{\prime}\right)\left(h_{*} \circ i\right) \leq G^{\prime}(h)$. That is, $F_{r^{\prime}\left(G^{\prime}\right)}^{-} \leq$ $G^{\prime}$, i.e., $G^{\prime} \in \operatorname{CCoeur}_{1}\left(F_{r^{\prime}\left(G^{\prime}\right)}^{-}\right)=\varsigma^{\prime}\left(r^{\prime}\left(G^{\prime}\right)\right)$.

Apply this to finite posets. Let $<$ be the strict part of $\leq$.

Definition 6.2 (Path Space) Let $Y$ be any finite pointed poset. Write $y \rightarrow y^{\prime}$ iff $y$ is immediately below $y^{\prime}$, i.e., $y<y^{\prime}$, and there is no $z \in Y$ such that $y<z<y^{\prime}$. A path $\pi$ in $Y$ is any set $\left\{y_{0}, y_{1}, \ldots, y_{n}\right\} \subseteq Y$ with $y_{0}=\perp \rightarrow$ $y_{1} \rightarrow \ldots \rightarrow y_{n}$. The path space $\Pi(Y)$ is the set of paths in $Y$, ordered by $\subseteq$.

Alternatively, the ordering on paths $y_{0} \rightarrow y_{1} \rightarrow \ldots \rightarrow y_{n}$ is the prefix ordering on sequences $y_{0} y_{1} \ldots y_{n}$.

Lemma 6.3 Every finite pointed poset $Y$ is a quasiprojection of its path space $\Pi(Y)$.
Proof. See Figure 4, which displays the path space of the space $Y$ of Figure $2(i)$. Each gray region is labeled with an element from $Y$, which is the image by $r$ of every point in the region; e.g., the top right, 5 -element region is mapped to $j$ in $Y$. Conversely, $\varsigma$ maps each $y \in Y$ to the points in Figure 4 that are in the corresponding gray region or above.

Formally, let $X=\Pi(Y)$, and define $r: X \rightarrow Y$ by $r(\pi)=\max \pi$, i.e., $r\left(y_{0} \rightarrow y_{1} \rightarrow \ldots \rightarrow y_{n}\right)=y_{n}$. Conversely, for every $y \in Y$, let $\varsigma(y)$ be the set of all paths $\pi$ that go through $y$, i.e., such that $y \in \pi$. The maps $r$ and $\varsigma$ are vacuously continuous, $\varsigma(y)$ is trivially non-empty, compact, and saturated. Compute $\mathcal{Q} r(\varsigma(y))$ : this is the set of elements $z$ that are above $y_{n}$, for some path $y_{0} \rightarrow y_{1} \rightarrow$ $\ldots \rightarrow y_{n}$ going through $y$. So $\mathcal{Q} r(\varsigma(y))=\uparrow y$. Conversely, it is clear that $\pi \in \varsigma(r(\pi))$ for every path $\pi$.

$Y$ is certainly not a retract of $\Pi(Y)$ in general: it is, iff $Y$ is a tree, i.e., a finite pointed poset where $\downarrow y$ is totally ordered for every $y \in Y$ (if $Y$ is a tree, then $Y \cong \Pi(Y)$, conversely $\Pi(Y)$ is a tree, and every retract of a tree is a tree).

Proposition 6.4 For every finite pointed poset $Y, \mathbf{V}_{1}(Y)$ is a continuous $\omega \mathbf{Q R B}-$ domain.

Proof. $\quad Y$ is trivially a continuous pointed depo. Then we know that $\mathbf{V}_{1}(Y)$ is again continuous [6, Section 3], and that $\mathbf{V}_{1}(Y)=\mathbf{V}_{1 w k}(Y)$ by the Kirch-Tix Theorem. Similarly for $\mathbf{V}_{1}(\Pi(Y))$. $\Pi(Y)$ is clearly stably compact, since finite. By Theorem 6.1, using Lemma 6.3, $\mathbf{V}_{1}(Y)$ is a quasi-projection of $\mathbf{V}_{1}(\Pi(Y))$. But $\Pi(Y)$ is a tree, so $\mathbf{V}_{1}(\Pi(Y))$ is an RB-domain, after Jung and Tix [16, Theorem 13]. Their proof actually exhibits a generating sequence of deflations. So $\mathbf{V}_{1}(\Pi(Y))$ is an $\omega \mathbf{Q R B}$-domain. We also know that $\mathbf{V}_{1}(Y)$ is stably compact, since $Y$ is [2]. So $\mathbf{V}_{1}(Y)$ is an $\omega \mathbf{Q R B}$-domain, by Proposition 4.3.

We can finally prove the main theorem of this paper.

Theorem 6.5 The probabilistic powerdomain of any $\omega \mathbf{Q R B}$-domain is an $\omega \mathbf{Q R B}$-domain.

Proof. Let $Y$ be an $\omega$ QRB-domain. By Theorem 4.6, $Y$ is a quasi-projection of some bifinite domain $X=\lim _{i \in \mathbb{N}} X_{i}$. Since $\mathbf{V}_{1}$ is a locally continuous functor on the category of dcpos, $\mathbf{V}_{1}(X)$ is also a bilimit of the spaces $\mathbf{V}_{1}\left(X_{i}\right), i \in I$. (E.g., this is mentioned in [16, Lemma 11].) Each $\mathbf{V}_{1}\left(X_{i}\right)$ is a continuous $\omega$ QRB-domain by Proposition 6.4, hence so is $\mathbf{V}_{1}(X)$, by Theorem 5.3 and since bilimits of continuous dcpos are continuous [1, Theorem 3.3.11].

Since $X$ is bifinite, it is stably compact, and $\mathbf{V}_{1}(X)=$ $\mathbf{V}_{1 w k}(X)$ because $X$ is continuous and pointed, using the Kirch-Tix Theorem. So $\mathbf{V}_{1 w k}(Y)$ is a quasi-projection of $\mathbf{V}_{1}(X)$ by Theorem 6.1. Since $Y$ is stably compact (Theorem 3.9), $\mathbf{V}_{1 w k}(Y)$ is stably compact [2], and is in particular sober, hence a well-filtered monotone convergence 
space [1, Corollary 7.2.11, Proposition 7.2.13]. So Proposition 4.3 applies: $\left(\mathbf{V}_{1 w k}(Y)\right)_{\sigma}$ is an $\omega \mathbf{Q R B}$-domain.

It is easy to see that the specialization quasi-ordering $\preceq$ of $\mathbf{V}_{1 w k}(Y)$ is the usual ordering on $\mathbf{V}_{1}(Y)$, i.e., $\nu \preceq \nu^{\prime}$ iff $\nu(U) \leq \nu^{\prime}(U)$ for every open $U$ of $Y$ (note that if $\nu \preceq \nu^{\prime}$, then $\nu^{\prime} \in[U>r]$ for every $\left.r<\nu(U)\right)$.

So $\left(\mathbf{V}_{1 w k}(Y)\right)_{\sigma}=\mathbf{V}_{1}(Y)$, and we conclude.

Using the fact that $\mathbf{V}_{1}(X)$ is continuous whenever $X$ is continuous and pointed [6, Section 3$]$, it also follows:

Corollary 6.6 The probabilistic powerdomain of any continuous $\omega \mathbf{Q R B}$-domain (in particular, every RB-domain) is again a continuous $\omega \mathbf{Q R B}-d o m a i n$.

\section{Conclusion, Failures and Perspectives}

We have shown that the category $\omega \mathbf{Q R B}$ of $\omega \mathbf{Q R B}$ domains and continuous maps was a category of quasicontinuous, stably compact dcpos that is closed, not only under finite products, bilimits, retracts (and even quasiretracts), but also under the probabilistic powerdomain functor $\mathbf{V}_{1}$. It is thus reasonably well-behaved.

But $\omega \mathbf{Q R B}$ is not cartesian-closed: as the anonymous referees (which I thank heartily) have noticed, the space $T$ of [1, Figure 12] is an $\omega \mathbf{Q R B}$-domain such that $[T \rightarrow T]$ is not even Lawson-compact, so not an $\omega$ QRB-domain. This also shows that, although $T$ is both continuous (even algebraic) and an $\omega \mathbf{Q R B}$-domain, $T$ is not an RB-domain: so Corollary 6.6 is not enough to settle the Jung-Tix problem in the positive either.

Shifting the focus towards the Kleisli category $\omega \mathbf{Q R B} \mathbf{B}_{\mathcal{Q}}$, for example, may be needed. This is a full subcategory of Jung, Kegelmann and Moshier's pleasing category $S C S^{*}$ of stably compact spaces and closed relations [15]. We plan to explore this in the future.

\section{References}

[1] S. Abramsky and A. Jung. Domain theory. In S. Abramsky, D. M. Gabbay, and T. S. E. Maibaum, editors, Handbook of Logic in Computer Science, volume 3, pages 1-168. Oxford University Press, 1994.

[2] M. Alvarez-Manilla, A. Jung, and K. Keimel. The probabilistic powerdomain for stably compact spaces. Theoretical Computer Science, 328(3):221-244, 2004.

[3] I. Battenfeld, M. Schröder, and A. Simpson. Compactly generated domain theory. Math. Struct. Computer Science, 16:141-161, 2006. Klaus Keimel Festschrift.

[4] I. Battenfeld, M. Schröder, and A. Simpson. A convenient category of domains. ENTCS, 172:69-99, 2007. Computation, Meaning and Logic, Articles dedicated to G. Plotkin.

[5] I. Battenfeld and A. Simpson. Two probabilistic powerdomains in topological domain theory. Domains IX Workshop, Sussex, UK, Sept. 2009.
[6] A. Edalat. Domain theory and integration. Theoretical Computer Science, 151:163-193, 1995.

[7] G. Gierz, K. H. Hofmann, K. Keimel, J. D. Lawson, M. Mislove, and D. S. Scott. Continuous lattices and domains. In Encyclopedia of Mathematics and its Applications, volume 93. Cambridge University Press, 2003.

[8] G. Gierz, J. D. Lawson, and A. Stralka. Quasicontinuous posets. Houston J. Math., 9:191-208, 1983.

[9] J. Goubault-Larrecq. Continuous capacities on continuous state spaces. In L. Arge, Ch. Cachin, T. Jurdziński, and A. Tarlecki, editors, Proc. 34th ICALP, pages 764-776. Springer-Verlag LNCS 4596, July 2007.

[10] J. Goubault-Larrecq. Continuous previsions. In J. Duparc and T. A. Henzinger, editors, Proc. 16th CSL, pages 542557. Springer-Verlag LNCS 4646, Sept. 2007.

[11] J. Goubault-Larrecq. De Groot duality and models of choice: Angels, demons, and nature. Math. Struct. Computer Science, 20:169-237, 2010.

[12] S. Graham. Closure properties of a probabilistic powerdomain construction. In M. Main, A. Melton, M. Mislove, and D. Schmidt, editors, 3rd MFPS Workshop, pages 213-233. Springer Verlag LNCS 298, 1988.

[13] C. Jones and G. D. Plotkin. A probabilistic powerdomain of evaluations. In Proc. 4th LICS, pages 186-195. IEEE, 1989.

[14] A. Jung. Cartesian Closed Categories of Domains. PhD thesis, Technische Hochschule Darmstadt, July 1998.

[15] A. Jung, M. Kegelmann, and M. A. Moshier. Stably compact spaces and closed relations. ENTCS, 45, 2001.

[16] A. Jung and R. Tix. The troublesome probabilistic powerdomain. In A. Edalat, A. Jung, K. Keimel, and M. Kwiatkowska, editors, Proc. Comprox III Workshop. ENTCS, 13:70-91, 1998.

[17] K. Keimel. Topological cones: Foundations for a domaintheoretical semantics combining probability and nondeterminism. ENTCS, 155:423-443, 2006.

[18] O. Kirch. Bereiche und Bewertungen. Master's thesis, Technische Hochschule Darmstadt, June 1993.

[19] J. D. Lawson. The versatile continuous order. In M. G. Main, A. Melton, M. W. Mislove, and D. A. Schmidt, editors, Proc. 3rd MFPS Workshop, pages 134-160. Springer Verlag LNCS 298, 1987.

[20] M. Mislove. Topology, domain theory and theoretical computer science. Topology and Its Applications, 89:3-59, 1998.

[21] R. Tix. Stetige Bewertungen auf topologischen Räumen. Diplomarbeit, TH Darmstadt, June 1995. 


\section{A Quasi-retracts of Stably Compact Spaces}

The purpose of the rest of this section is to show that, when $X$ is stably compact, and $Y$ is not only a quasi-retract of $X$ but a quasi-projection of $X$, then $Y$ is also stably compact. Since every QRB-domain is stably compact, $Y$ will be stably compact, hence sober, in this case, and the assumption that $Y_{\sigma}$ be a well-filtered monotone convergence space will be satisfied for free.

Before we embark on showing that the quasi-projection of every stably compact space is stably compact, we recall that no such subtlety would be needed in the classical case of retracts of continuous dcpos: any topological retract of a continuous dcpo is in fact a continuous dcpo, and in particular, has the Scott topology.

In all proofs of this section, let $r: X \rightarrow Y$ be a quasiretraction, with matching quasi-section $\varsigma: Y \rightarrow \mathcal{Q}(X)$.

Lemma A.1 Every quasi-retract $Y$ of a compact space $Y$ is compact.

Proof. The image of a compact set by a continuous map is compact. Now apply Lemma 4.2.

Define $\varsigma^{\prime}: \mathcal{Q}(Y) \rightarrow \mathcal{Q}(X)$ by $\varsigma^{\prime}(Q)=\bigcup_{y \in Q} \varsigma(y)$. Then $\varsigma^{\prime}$ is well-defined, and continuous. This is folklore. For completeness, here is a proof. First, $\varsigma^{\prime}(Q)$ is trivially saturated for any $Q \in \mathcal{Q}(Y)$. To show that it is compact, observe that a subset $K$ is compact iff in every directed cover of $K$ by opens, one of the opens of the cover already contains $K$; realize also that $\square$ commutes with directed unions; if $\varsigma^{\prime}(Q) \subseteq \bigcup_{j \in J}^{\uparrow} U_{j}$, then $Q \subseteq \varsigma^{-1}\left(\square \bigcup_{j \in J}^{\uparrow} U_{j}\right)=$ $\varsigma^{-1}\left(\bigcup_{j \in J}^{\uparrow} \square U_{j}\right)=\bigcup_{j \in J}^{\uparrow} \varsigma^{-1}\left(\square U_{j}\right)$, so $Q \subseteq \varsigma^{-1}\left(\square U_{j}\right)$ for some $j \in J$, i.e., $\varsigma^{\prime}(Q) \subseteq U_{j}$. Then for every subbasic open $\square U, U$ open in $X, \varsigma^{\prime-1}(\square U)=\square \varsigma^{-1}(\square U)$, so $\varsigma^{\prime}$ is continuous.

Lemma A.2 Any quasi-retract $Y$ of a well-filtered space $X$ is well-filtered.

Proof. Let $\left(Q_{i}\right)_{i \in I}$ be a filtered family of compact saturated subsets of $Y$, and assume that $\bigcap_{i \in I}^{\downarrow} Q_{i} \subseteq V$, where $V$ is open in $Y$. Let $Q_{i}^{\prime}=\varsigma^{\prime}\left(Q_{i}\right)$. This is compact saturated, and forms a directed family, since $\varsigma^{\prime}$ is monotonic. We claim that $\bigcap_{i \in I} Q_{i}^{\prime} \subseteq r^{-1}(V)$. Indeed, every $x \in \bigcap_{i \in I} Q_{i}^{\prime}$ is such that, for every $i \in I$, there is a $y_{i} \in Q_{i}$ such that $x \in \varsigma\left(y_{i}\right)$; then $r(x) \in \mathcal{Q} r\left(\varsigma\left(y_{i}\right)\right)=\uparrow y_{i}$, so $r(x) \in Q_{i}$, for every $i \in I$. Since $\bigcap_{i \in I}^{\downarrow} Q_{i} \subseteq V, r(x)$ is in $V$, whence the claim.

Since $X$ is well-filtered, $Q_{i}^{\prime} \subseteq r^{-1}(V)$ for some $i \in I$. Then, for every $y \in Q_{i}, \varsigma(y) \subseteq \varsigma^{\prime}\left(Q_{i}\right)=Q_{i}^{\prime} \subseteq r^{-1}(V)$, so $y \in \mathcal{Q} r(\varsigma(y)) \subseteq \mathcal{Q} r\left(r^{-1}(V)\right) \subseteq V$. So $Q_{i} \subseteq V$.

Now notice that $\mathcal{Q} r \circ \varsigma^{\prime}$ is the identity on $\mathcal{Q}(Y)$. Indeed, for every $Q \in \mathcal{Q}(Y), \mathcal{Q} r\left(\varsigma^{\prime}(Q)\right)=\uparrow\{r(x) \mid$ $\left.x \in \bigcup_{y \in Q} \varsigma(y)\right\}=\uparrow \bigcup_{y \in Q}\{r(x) \mid x \in \varsigma(y)\}=$ $\uparrow \bigcup_{y \in Q} \mathcal{Q} r(\varsigma(y))=\uparrow \bigcup_{y \in Q} \uparrow y=Q$.

Lemma A.3 Any quasi-retract $Y$ of a coherent space $X$ is coherent.

Proof. Let $Q_{1}, Q_{2}$ be two compact saturated subsets of $Y$. Then $\varsigma^{\prime}\left(Q_{1}\right) \cap \varsigma^{\prime}\left(Q_{2}\right)$ is compact saturated in $X$, using the fact that $X$ is coherent. So $\mathcal{Q} r\left(\varsigma^{\prime}\left(Q_{1}\right) \cap \varsigma^{\prime}\left(Q_{2}\right)\right)$ is compact saturated in $Y$. We claim that $\mathcal{Q} r\left(\varsigma^{\prime}\left(Q_{1}\right) \cap \varsigma^{\prime}\left(Q_{2}\right)\right)=$ $Q_{1} \cap Q_{2}$, which will finish the proof. In one direction, every element $y$ of $Q_{1} \cap Q_{2}$ is in $\mathcal{Q} r\left(\varsigma^{\prime}\left(Q_{1}\right) \cap \varsigma^{\prime}\left(Q_{2}\right)\right)$ : by Lemma 4.2, pick $x$ such that $y=r(x)$, and observe that $x \in \varsigma^{\prime}\left(Q_{1}\right)$ (indeed $x \in \varsigma(y)$, where $\left.y \in Q_{1}\right)$ and $x \in \varsigma^{\prime}\left(Q_{2}\right)$. In the other direction, $\mathcal{Q} r\left(\varsigma^{\prime}\left(Q_{1}\right) \cap \varsigma^{\prime}\left(Q_{2}\right)\right) \subseteq$ $\mathcal{Q}(r)\left(\varsigma^{\prime}\left(Q_{1}\right)\right) \cap \mathcal{Q}(r)\left(\varsigma^{\prime}\left(Q_{2}\right)\right)=Q_{1} \cap Q_{2}$, since $\mathcal{Q} r \circ \varsigma^{\prime}$ is the identity on $\mathcal{Q}(Y)$.

Notice that, for every open subset $V$ of $Y, V=$ $\varsigma^{-1}\left(\square r^{-1}(V)\right)$; indeed, $y \in \varsigma^{-1}\left(\square r^{-1}(V)\right)$ iff $\varsigma(y) \in$ $\square r^{-1}(V)$, iff $\varsigma(y) \subseteq r^{-1}(V)$, iff $\mathcal{Q} r(\varsigma(y)) \subseteq V$, iff $\uparrow y \subseteq V$.

Lemma A.4 Any quasi-projection $Y$ of a locally compact space $X$ is locally compact.

Proof. Let $V$ be any open subset of $Y$, and $y \in V$. Then $y \in \varsigma^{-1}\left(\square r^{-1}(V)\right)$, i.e., $\varsigma(y) \subseteq r^{-1}(V)$. Since $X$ is locally compact, whenever $Q$ is compact and $U$ is open in $X$ and $Q \subseteq U$, there is a compact saturated subset $Q^{\prime}$ of $X$ such that $Q \subseteq \operatorname{int}\left(Q^{\prime}\right)$ and $Q^{\prime} \subseteq U$. (This is well-known: by local compactness, there is a compact saturated subset $Q_{x}$ such that $x \in \operatorname{int}\left(Q_{x}\right)$ and $Q_{x} \subseteq U$ for each $x \in Q$; since $Q \subseteq \bigcup_{x \in Q} \operatorname{int}\left(Q_{x}\right)$, by compactness there is a finite subset $E$ of $Q$ such that $Q \subseteq \bigcup_{x \in E} \operatorname{int}\left(Q_{x}\right)$. Then take $Q^{\prime}=\bigcup_{x \in E} Q_{x}$.)

Take $Q=\varsigma(y), U=r^{-1}(V)$, and pick $Q^{\prime}$ as above, i.e., $\varsigma(y) \subseteq \operatorname{int}\left(Q^{\prime}\right)$ and $Q^{\prime} \subseteq r^{-1}(V)$. Let $Q^{\prime \prime}=\mathcal{Q} r\left(Q^{\prime}\right)$, so that $Q^{\prime \prime}$ is compact saturated in $Y$ and $Q^{\prime \prime} \subseteq V$.

We claim that, since $r$ is a quasi-projection, $r^{-1}\left(\varsigma^{-1}(\square U)\right) \subseteq U$ for every open subset $U$ of $X$. Indeed, for every $x \in r^{-1}\left(\varsigma^{-1}(\square U)\right), \varsigma(r(x)) \subseteq U$. Since $r$ is a quasi-projection, $x \in \varsigma(r(x))$, so $x \in U$. So the open subset $W=\varsigma^{-1}\left(\square \operatorname{int}\left(Q^{\prime}\right)\right)$ of $Y$ is such that $r^{-1}(W) \subseteq \operatorname{int}\left(Q^{\prime}\right)$. In particular, $W$ is included in the image of $Q^{\prime}$ by $r$, hence in $Q^{\prime \prime}$. Also, $y \in W$, since $\varsigma(y) \subseteq \operatorname{int}\left(Q^{\prime}\right)$. So $y$ is included in an open subset $W$ of $Q^{\prime \prime}$, hence in $\operatorname{int}\left(Q^{\prime \prime}\right)$.

Recall that a space is $T_{0}$ iff its specialization quasiordering $\leq$ is an ordering, i.e., $y \leq y^{\prime}$ and $y^{\prime} \leq y$ imply $y=y^{\prime}$. In general, a quasi-projection of a $T_{0}$ space may fail to be $T_{0}$. E.g., take $X=\{a, b\}$ with its discrete topology, $Y=\{c, d\}$ with the topology containing only $\emptyset$ and $Y$ itself, $r(a)=r(b)=c, \varsigma(c)=\varsigma(d)=\{a, b\}$. Note that $\uparrow c=\uparrow d=Y$ in $Y$. 
However, putting together the results in this Appendix, we obtain:

Proposition 4.4: Every $T_{0}$ quasi-projection $Y$ of a stably compact space $X$ is stably compact.

Proof. $\quad Y$ is $T_{0}$ by assumption, and locally compact, well-filtered, compact, and coherent. In the presence of local compactness, $T_{0}$ and well-filteredness are equivalent to sobriety [7, Theorem II-1.21], whence the claim.

\section{B Proof of Lemma 5.1}

Proof. Let $\left(\varphi_{i}\right)_{i \in I}$ be a directed family of quasideflations with $\uparrow x=\bigcap_{i \in I}^{\downarrow} \uparrow \varphi_{i}(x)$ for all $x \in X$, and $\left(\psi_{j}\right)_{j \in J}$ a directed family of quasi-deflations with $\uparrow y=\bigcap_{j \in J}^{\downarrow} \uparrow \psi_{j}(y)$ for all $y \in Y$. Consider the maps $\chi_{i j}$, sending $(x, y)$ to $\varphi_{i}(x) \times \psi_{j}(y)$. Clearly, $(x, y) \in \uparrow \chi_{i j}(x, y)$, and $\operatorname{Im} \chi_{i j}$ is finite. For all $i, j, \quad \chi_{i j}$ is easily seen to be Scott-continuous, and $\bigcap_{i \in I, j \in J}^{\downarrow} \uparrow \chi_{i j}(x, y)=\bigcap_{i \in I, j \in J}^{\downarrow} \uparrow\left(\varphi_{i}(x) \times \psi_{j}(y)\right)=$ $\bigcap_{i \in I}^{\downarrow} \uparrow \varphi_{i}(x) \times \bigcap_{j \in J}^{\downarrow} \uparrow \psi_{j}(y)=\uparrow x \times \uparrow y=\uparrow(x, y)$. The case of $\omega \mathbf{Q R B}$-domains is similar.

\section{Proof of Theorem 5.3}

Proposition 5.2: Let $X$ be a poset. Then $X$ is a QRBdomain iff $X$ is a quasi-continuous depo and the set $G$ of quasi-deflations on $X$ is qfs.

Proof. If $X$ is a QRB-domain, then let $\left(E_{k}, x_{k}\right) \in$ $\operatorname{Fin}(X) \times X$, be such that $E_{k} \ll x_{k}$ for every $k, 1 \leq k \leq n$, and $\left(\varphi_{i}\right)_{i \in I}$ be a generating family of quasi-deflations. For each $k, 1 \leq k \leq n, x_{k}=\uparrow x_{k}=\bigcap_{i \in I}^{\downarrow} \uparrow \varphi_{i}\left(x_{k}\right) \subseteq \uparrow E_{k}$, so by Proposition 3.4 there is an $i \in I$ such that $\uparrow \varphi_{i}\left(x_{k}\right) \subseteq$ $\uparrow E_{k} \subseteq \uparrow E_{k}$. And we may pick the same $i$ for every $k$, by directedness. So $\varphi_{i}$ is the desired $g \in G$.

Conversely, assume that $G$ is qfs. We show that $H=$ $\left\{\varphi^{\dagger} \circ \varphi \mid \varphi \in G\right\}$ is a generating family of quasideflations. First, for each $\varphi \in G, \varphi^{\dagger} \circ \varphi$ is continuous by Lemma 3.6. Since $x \in \uparrow \varphi(x)$, pick $x^{\prime} \in \varphi(x)$ such that $x^{\prime} \leq x$. Then $x \in \uparrow \varphi\left(x^{\prime}\right)$, since $\varphi\left(x^{\prime}\right) \leq \sharp \varphi(x)$. So $x \in \bigcup_{x^{\prime} \in \varphi(x)} \uparrow \varphi\left(x^{\prime}\right)=\varphi^{\dagger} \circ \varphi(x)$. So $\varphi^{\dagger} \circ \varphi$ is a quasideflation.

Let us show that $H$ is directed. Pick $\varphi$ and $\varphi^{\prime}$ from $G$. Let $E=\operatorname{Im} \varphi, E^{\prime}=\operatorname{Im} \varphi^{\prime}$. For each $y \in E, \varphi(y) \ll y$ by Lemma 3.2. Since $X$ is quasi-continuous, use interpolation, and pick a finite set $E_{y}$ such that $\varphi(y) \ll E_{y} \ll y$. Similarly, let $E_{y^{\prime}}^{\prime}$ be a finite set such that $E_{y^{\prime}}^{\prime} \ll y^{\prime}$ and $\varphi^{\prime}\left(y^{\prime}\right) \ll E_{y^{\prime}}^{\prime}$ for each $y^{\prime} \in E^{\prime}$.

Consider the finite collection of all pairs $\left(E_{y}, y\right)$, $(\varphi(y), z),\left(E_{y^{\prime}}^{\prime}, y^{\prime}\right)$, and $\left(\varphi^{\prime}\left(y^{\prime}\right), z^{\prime}\right)$, where $y \in M, z \in$ $E_{y}, y^{\prime} \in E^{\prime}, z^{\prime} \in E_{y^{\prime}}$. Since $G$ is qfs, there is a $\psi \in G$ such that $E \leq \sharp \psi(x) \leq \sharp\{x\}$ for all the above pairs
$(E, x)$. In particular, looking at the pair $\left(E_{y}, y\right)$, we get: (a) $E_{y} \leq^{\sharp} \psi(y)$ for every $y \in E$. And looking at the pair $(\varphi(y), z), \varphi(y) \leq^{\sharp} \psi(z)$ for all $y \in E, z \in E_{y}$. It follows: (b) $\varphi(y) \leq \sharp \psi^{\dagger}\left(E_{y}\right)$ for every $y \in E$. Then, for every $x \in X, \varphi^{\dagger} \circ \varphi(x)=\bigcup_{y \in \varphi(x)} \varphi(y) \leq \sharp \bigcup_{y \in \varphi(x)} \psi^{\dagger}\left(E_{y}\right)$ (by $(b)) \leq \sharp \bigcup_{y \in \varphi(x)} \psi^{\dagger} \circ \psi(y)$ (by $\left.a\right)=\psi^{\dagger} \circ \psi^{\dagger} \circ \varphi(x) \leq \sharp$ $\psi^{\dagger} \circ \psi^{\dagger}(\{x\})($ since $\varphi(x) \leq \sharp\{x\})=\psi^{\dagger} \circ \psi(x)$. So $\varphi^{\dagger} \circ \varphi$ is below $\psi^{\dagger} \circ \psi$. Similarly, $\varphi^{\prime \dagger} \circ \varphi^{\prime}$ is below $\psi^{\dagger} \circ \psi$, so $H$ is directed.

Finally, we claim that $\bigcap_{\varphi \in G} \uparrow \varphi^{\dagger} \circ \varphi(x)=\uparrow x$. In the $\supseteq$ direction, this is because $\varphi^{\dagger} \circ \varphi$ is a quasi-retraction. Conversely, let $E \in \operatorname{Fin}(X)$ be such that $E \ll x$. By interpolation, find $E^{\prime} \in \operatorname{Fin}(X)$ such that $E \ll E^{\prime} \ll x$. Since $G$ is qfs, applied to the pairs $\left(E^{\prime}, x\right)$ and $(E, y)$ for each $y \in E^{\prime}$, there is an element $\varphi \in G$ such that $E^{\prime} \leq \sharp \varphi(x)$ and $E \leq \sharp$ $\varphi(y)$ for every $y \in E^{\prime}$. So $E \leq \sharp \varphi^{\dagger}\left(E^{\prime}\right) \leq \sharp \varphi^{\dagger} \circ \varphi(x)$. So $\bigcap_{\varphi \in G} \uparrow \varphi^{\dagger} \circ \varphi(x) \subseteq \bigcap_{E \in \operatorname{Fin}(X), E \ll x}^{\downarrow} \uparrow E=\uparrow x$, as $X$ is quasi-continuous.

Theorem 5.3: Any bilimit of $\omega \mathbf{Q R B}$-domains is an $\omega$ QRB-domain.

Proof. Let $\left(X_{i}\right)_{i \in \mathbb{N}}$ be an expanding sequence of QRBdomains, with projections $p_{i j}: X_{j} \rightarrow X_{i}$ and embeddings $e_{i j}: X_{i} \rightarrow X_{j}, i \leq j$. Let $X=\lim _{i \in \mathbb{N}} X_{i}$. There is a projection $p_{i}: X \rightarrow X_{i}$, given by $p_{i}(\vec{x})=x_{i}$, and an embedding $e_{i}: X_{i} \rightarrow X$ for every $i \in \mathbb{N}$.

For each map $f: Y \rightarrow Z$, let $\operatorname{Fin} f: \operatorname{Fin}(Y) \rightarrow \operatorname{Fin}(Z)$ map $E \in \operatorname{Fin}(Y)$ to its image by $f$. We observe that if $E \ll p_{i}(\vec{x})$ in $X_{i}$, then Fin $e_{i j}(E) \ll p_{j}(\vec{x})$ for every $j \geq i$. Indeed, consider any directed family $\left(y_{k}\right)_{k \in I}$ such that $p_{j}(\vec{x}) \leq \sup _{k \in I} y_{k}$. Then $p_{i}(\vec{x})=p_{i j}\left(p_{j}(\vec{x})\right) \leq$ $\sup _{k \in I} p_{i j}\left(y_{k}\right)$, so for some $k \in I$, there is a $z \in E$ with $z \leq p_{i j}\left(y_{k}\right)$. Then $e_{i j}(z) \leq e_{i j}\left(p_{i j}\left(y_{k}\right)\right) \leq y_{k}$. We conclude since $e_{i j}(z) \in$ Fin $e_{i j}(E)$.

It follows that the family $\mathcal{D}_{\vec{x}}$ of all finite subsets of the form Fin $e_{i}(E)$, where $E \in \operatorname{Fin}\left(X_{i}\right)$ and $E \ll p_{i}(\vec{x})$, $i \in \mathbb{N}$, is directed. Indeed, given Fin $e_{i}(E)$ and Fin $e_{j}\left(E^{\prime}\right)$ in $\mathcal{D}_{\vec{x}}$, we can first assume that $i=j$. Otherwise, say if $j \geq i$, then Fin $e_{i}(E)$ is also equal to Fin $e_{j}\left(\right.$ Fin $\left.e_{i j}(E)\right)$, and by $(a)$ Fin $e_{i j}(E) \ll p_{j}(\vec{x})$. Then, since $X_{i}$ is quasicontinuous, there is an $E^{\prime \prime} \in \operatorname{Fin}\left(X_{i}\right)$ such that $E, E^{\prime} \leq \sharp$ $E^{\prime \prime} \ll p_{i}(\vec{x})$, and we are done.

Moreover, $\bigcap_{\text {Fin } e_{i}(E) \in \mathcal{D}_{\vec{x}}} \uparrow$ Fin $e_{i}(E)$ equals $\uparrow \vec{x}$. That it contains $x$ is obvious: whenever $E \ll p_{i}(\vec{x})$, pick $z \in E$ with $z \leq p_{i}(\vec{x})$, so that $e_{i}(z) \leq e_{i}\left(p_{i}(\vec{x})\right) \leq$ $\vec{x}$, hence $\vec{x} \in \uparrow$ Fin $e_{i}(E)$. Conversely, every $\vec{z} \in$ $\bigcap_{\text {Fin } e_{i}(E) \in \mathcal{D}_{\vec{x}}} \uparrow$ Fin $e_{i}(E)$ must be such that $z_{i}=p_{i}(\vec{z}) \in$ $\bigcap_{E \ll p_{i}(\vec{x})} p_{i}\left(\uparrow\right.$ Fin $\left.e_{i}(E)\right)$. But $E \ll p_{i}(\vec{x})$ implies that some element of $E$ is below $p_{i}(\vec{x})$, hence that some element of Fin $e_{i}(E)$ is below $e_{i}\left(p_{i}(\vec{x})\right) \leq \vec{x}$; then $p_{i}\left(\uparrow\right.$ Fin $\left.e_{i}(E)\right)$ contains an element below $p_{i}(\vec{x})=x_{i}$ : so $x_{i} \leq z_{i}$. As this holds for every $i, \vec{x} \leq \vec{z}$. So $\bigcap_{\text {Fin } e_{i}(E) \in \mathcal{D}} \uparrow \operatorname{Fin} e_{i}(E) \subseteq \uparrow \vec{x}$.

In particular, $X$ is a quasi-continuous depo. 
We check that the set of quasi-deflations on $X$ is qfs. Consider a finite collection of pairs $\left(\vec{E}_{k}, \vec{x}_{k}\right) \in$ $\operatorname{Fin}(X) \times X$ with $\vec{E}_{k} \ll \vec{x}_{k}, 1 \leq k \leq n$. Since $\bigcap_{\text {Fin } e_{i}(E) \in \mathcal{D}_{\vec{x}_{k}}} \uparrow$ Fin $e_{i}(E)=\uparrow \vec{x}_{k}$, by Proposition 3.4, for each $k$, pick $i \in \mathbb{N}$ and $E_{k} \in \operatorname{Fin}\left(X_{i}\right)$ such that $E_{k} \ll$ $p_{i}\left(\vec{x}_{k}\right)$ and $\vec{E}_{k} \leq \sharp$ Fin $e_{i}\left(E_{k}\right)$. (We can pick the same $i$ for every $k$, as above.) Since $X_{i}$ is a QRB-domain, there is a quasi-deflation $\varphi$ on $X_{i}$ such that $\varphi\left(p_{i}\left(\vec{x}_{k}\right)\right) \subseteq \uparrow E_{k}$ for every $k, 1 \leq k \leq n$.

Consider $\psi: X \rightarrow \operatorname{Fin}(X)$ defined as Fin $e_{i} \circ \varphi \circ p_{i}$. Fin $e_{i}$, restricted to $\operatorname{Im} \varphi$, which is finite, is trivially Scottcontinuous. So $\psi$ is continuous. For every $\vec{x} \in X$, some element $y$ of $\varphi\left(p_{i}(\vec{x})\right)$ is below $p_{i}(\vec{x})$, since $\varphi$ is a quasideflation. Then $e_{i}(y)$ is below $e_{i}\left(p_{i}(\vec{x})\right) \leq \vec{x}$, and is in $\psi(\vec{x})$. So $\psi$ is a quasi-deflation.

Moreover, by construction, for each $k, 1 \leq k \leq n$, $\psi\left(\vec{x}_{k}\right) \subseteq \uparrow$ Fin $e_{i}\left(E_{k}\right)$, so $\psi\left(\vec{x}_{k}\right) \subseteq \uparrow \vec{E}_{k}$, since $\vec{E}_{k} \leq \sharp$ Fin $e_{i}\left(E_{k}\right)$. So the set of quasi-deflations on $X$ is qfs.

By Proposition 5.2, $X$ is then a QRB-domain. It is easy to see that any (countable) bilimit of countably-based quasicontinuous dcpos is countably-based. Indeed, a countably based quasi-continuous dcpo $X_{i}$ has a countable base of sets of the form $\uparrow E_{i k}, E \in \operatorname{Fin}\left(X_{i}\right), k \in \mathbb{N}$. The $\mathcal{D}_{\vec{x}}$ construction above, suitably modified, shows that the sets ^Fin $e_{i}\left(E_{i k}\right), i, k \in \mathbb{N}$, form a, necessarily countable, base of the topology on $X$. By Proposition 3.13, $X$ is an $\omega$ QRB-domain. 